\title{
Idaho National Laboratory Annual Report for Permit to Construct P-2015.0023 for Calendar Year 2018
}

March 2019

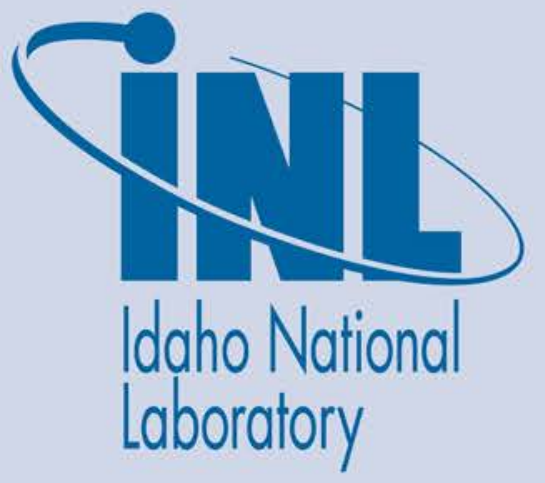

The INL is a U.S. Department of Energy National Laboratory operated by Battelle Energy Alliance 
INTENTIONALLY BLANK 


\section{Idaho National Laboratory Annual Report for Permit to Construct P-2015.0023 for Calendar Year 2018}

March 2019

Idaho National Laboratory Idaho Falls, Idaho 83415

http://www.inl.gov

Prepared for the U.S. Department of Energy Office of Nuclear Energy Under DOE Idaho Operations Office

Contract DE-AC07-05ID14517 
INTENTIONALLY BLANK 


\begin{abstract}
The U.S. Department of Energy (DOE) Idaho National Laboratory (INL) Site operates facilities with potential emissions of criteria and hazardous air pollutants.

This report documents the calendar year 2018 criteria and hazardous air pollutants emissions and has been prepared to comply with permit to construct (PTC) P-2015-0023, Condition 2.9 and Idaho Administrative Procedures Act (IDAPA) 58.01.01.178, Standard Contents of Permits Establishing a Facility Emissions Cap. IDAPA 58.01.01.178.04 requires that all permits establishing a FEC shall include sufficient reporting to assure compliance with the permit establishing the FEC.

Total hazardous air pollutant (HAP) emissions for the year were 1.49 tons in aggregate and the maximum individual HAP was hydrochloric acid at 1.26 tons. Criteria pollutant emissions were 2.16 tons for sulfur dioxide, 35.65 tons for oxides of nitrogen, 11.58 tons of carbon monoxide, 3.51 tons of total particulate matter and 2.66 tons of VOCs. No permitted limits were exceeded.
\end{abstract}


INTENTIONALLY BLANK 


\section{CONTENTS}

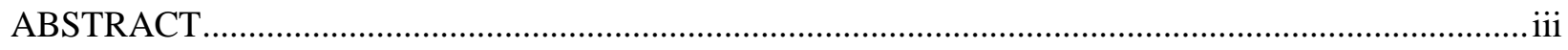

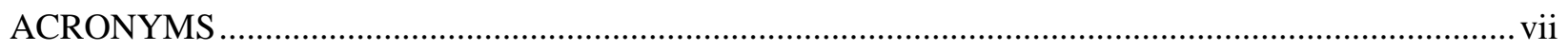

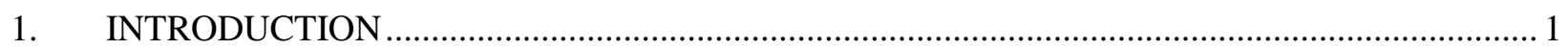

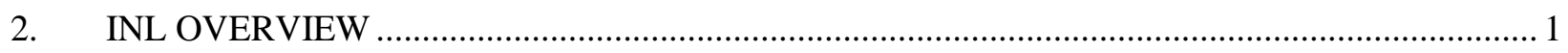

3. 2018 Criteria and Hazardous Air Pollutant Emissions ............................................................... 3

4. Monthly and 12 Month Rolling Emissions Totals.................................................................... 3

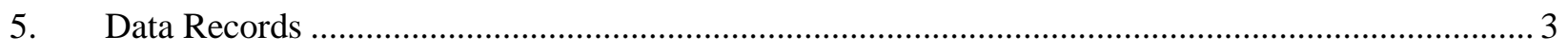

6. Methods, Equations and Emission Factors .............................................................................. 3

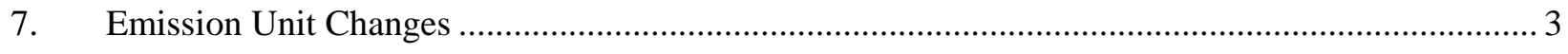

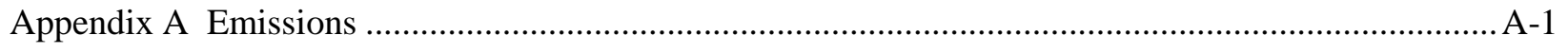

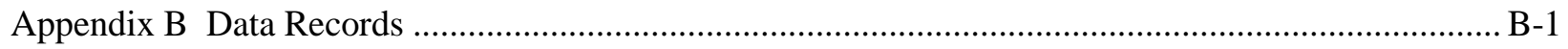

Appendix C Updated Equipment List.......................................................................................... C-1

\section{FIGURES}

Figure 1 - Idaho National Laboratory Site Facilities ...................................................................... 2

\section{TABLES}

Table 1 - CY-2018 Criteria and Hazardous Air Pollution Emissions ........................................................ 3

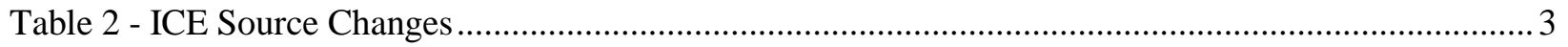

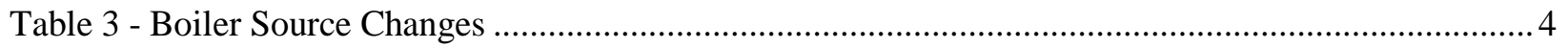


INTENTIONALLY BLANK 


\section{ACRONYMS}

AMWTP Advanced Mixed Waste Treatment Project

BEA Battelle Energy Alliance

CFA Central Facilities Area

CO carbon monoxide

DOE Department of Energy

ICE internal combustion engines

IDAPA a numbering designation for all administrative rules in Idaho promulgated in accordance with the Idaho Administrative Procedures Act

INL Idaho National Laboratory

HAP hazardous air pollutant

MFC Materials and Fuels Complex

$\mathrm{NO}_{2} \quad$ nitrogen dioxide

NRF Naval Reactors Facility

PM particulate matter

$\mathrm{PM}_{2.5} \quad$ particulate matter with an aerodynamic diameter less than or equal to a nominal 2.5 micrometers

$\mathrm{PM}_{10} \quad$ particulate matter with an aerodynamic diameter less than or equal to a nominal 10 micrometers

PTC permit to construct

RWMC Radioactive Waste Management Complex

SMC Specific Manufacturing Capability

$\mathrm{SO}_{2} \quad$ sulfur dioxide

TAN Test Area North

$\mathrm{T} / \mathrm{yr} \quad$ tons per consecutive 12 calendar month period

VOC volatile organic compounds 
INTENTIONALLY BLANK 


\section{Idaho National Laboratory Annual Report for Permit to Construct P-2015.0023 for Calendar Year 2018}

\section{INTRODUCTION}

The U.S. Department of Energy (DOE) Idaho National Laboratory (INL) Site operates facilities with potential emissions criteria pollutants and hazardous air pollutants (HAPs). This report has been prepared to comply with permit to construct (PTC) P-2015.0023, Condition 2.9 and Idaho Administrative Procedures Act (IDAPA) 58.01.01.178.04, Recordkeeping.

This report documents the criteria pollutants and hazardous air pollutant emissions from the INL emission sources regulated by PTC P-2015.0023. In addition to annual emissions the report includes record summaries of the data used for determining the 12-month total facility-wide criteria pollutant and HAP emissions, the 12-month rolling emissions totals generated under the criteria pollutant emissions calculation and HAP emissions calculation for the reporting period. A table of emissions units with changes that occurred during the calendar year is also included.

\section{INL OVERVIEW}

INL is a science-based, applied engineering national laboratory dedicated to supporting DOE's missions in nuclear and energy research, science, and national defense. Battelle Energy Alliance, LLC, (BEA) is the maintenance and operations contractor and operates INL under contract with DOE. BEA conducts research, development, demonstration, and deployment activities. INL also manufactures armor at the Specific Manufacturing Capability (SMC) facility for Abrams tanks under a government-togovernment contract with the Department of Defense. In addition to the primary mission of INL, environmental cleanup and remediation is also performed at INL by Fluor Idaho, LLC. The Naval Reactors Facility (NRF), operated for the U.S. Naval Nuclear Propulsion Program by the Fluor Marine Propulsion, LLC, prepares and packages spent naval nuclear fuel for dry storage and eventual transport to a permanent repository.

There are currently 7 major facility areas at the INL Site (Figure 1) that are potential sources of pollutant emissions that are included in this report. They are:

- $\quad$ Radioactive Waste Management Complex (RWMC) and the neighboring Advanced Mixed Waste Treatment Project (AMWTP),

- Advanced Test Reactor Complex,

- Central Facilities Area (CFA),

- Idaho Nuclear Technology and Engineering Center,

- Materials and Fuels Complex (MFC),

- NRF, and

- $\quad$ Test Area North (TAN) that includes the SMC facility. 


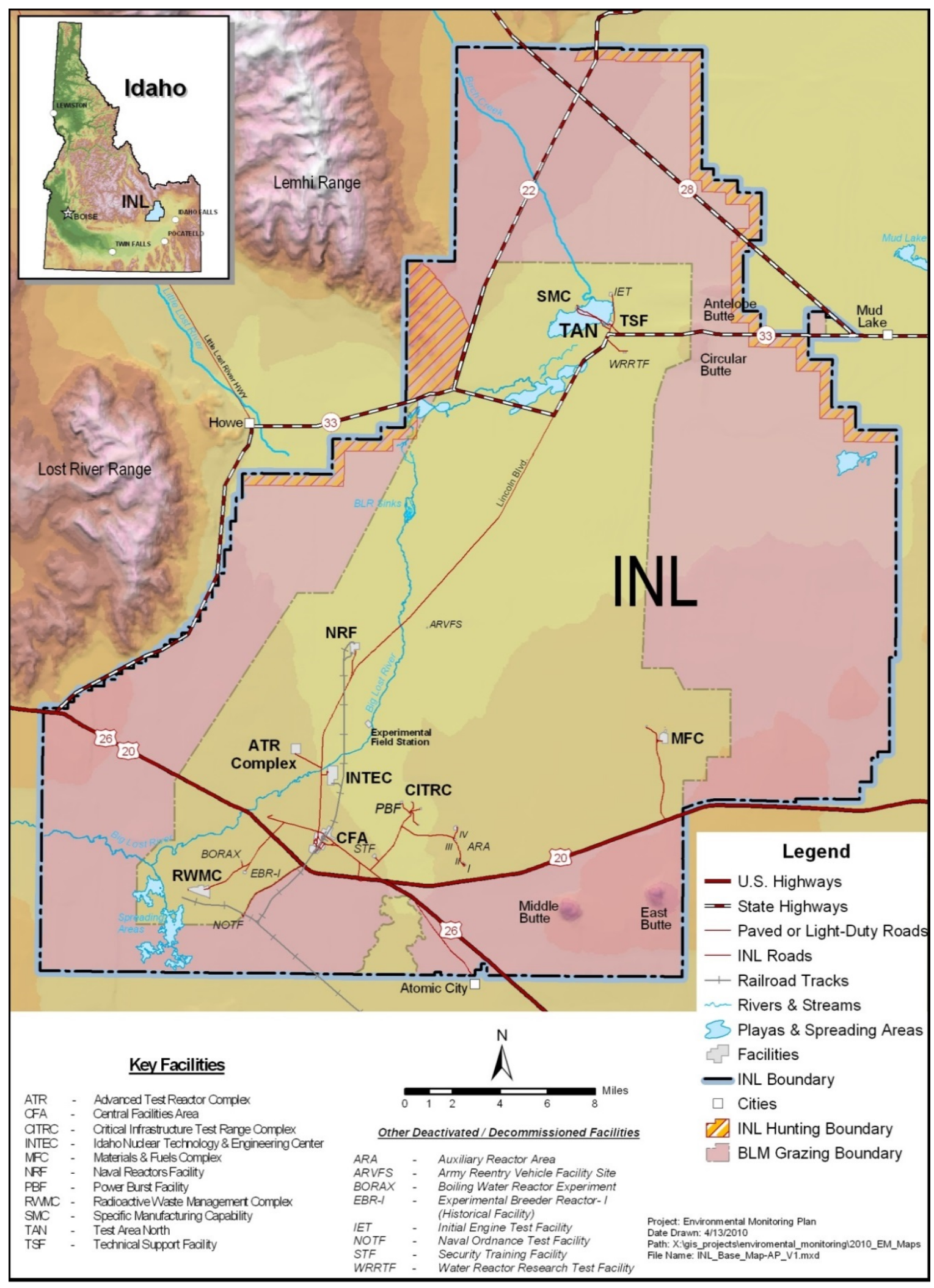

Figure 1 - Idaho National Laboratory Site Facilities 


\section{2018 Criteria and Hazardous Air Pollutant Emissions}

Facility-wide emissions from the US Department of Energy-INL facility are listed in the Table 1. Table 1 - CY-2018 Criteria and Hazardous Air Pollution Emissions

\begin{tabular}{|c|c|c|c|c|c|c|c|}
\hline $\mathbf{P M}_{10 / \mathbf{P M}}$ & SO $_{2}$ & NOx & CO & VOC & \multicolumn{2}{|c|}{ Individual HAP } & $\begin{array}{c}\text { Aggregate } \\
\text { HAP }\end{array}$ \\
\hline $\mathbf{T} / \mathbf{y r}$ & $\mathbf{T} / \mathbf{y r}$ & $\mathbf{T} / \mathbf{y r}$ & $\mathbf{T} / \mathbf{y r}$ & $\mathbf{T} / \mathbf{y r}$ & $\mathbf{T} / \mathbf{y r}$ & HAP & T/yr \\
\hline 3.51 & 2.16 & 35.65 & 11.58 & 2.66 & 1.26 & Hydrochloric Acid & 1.49 \\
\hline
\end{tabular}

\section{Monthly and 12 Month Rolling Emissions Totals}

Appendix A contains the monthly and 12-month rolling emissions totals generated under the criteria pollutant emissions calculation and HAP emissions calculation for the reporting period.

\section{Data Records}

Records of data used for determining emissions are contained in Appendix B and are summaries of records maintained at the individual facilities.

\section{Methods, Equations and Emission Factors}

No new emission methods, equations, emissions factors, or sources for emissions factors were used to determine the 12-month total facility-wide criteria pollutant and HAP emissions for the calendar year.

\section{Emission Unit Changes}

The following emission unit changes occurred in calendar year 2018.

Nine new emergency internal combustion engines (ICE) were installed at INL after the application for PTC P-2015.0023 was submitted and two ICE were removed from service and replaced by engines meeting the requirements of 40 CFR Part 60 Subpart IIII, for compression ignition engines. Five engines that were inadvertently omitted from the permit application. See Table 2 below. All changes have been documented in the list of stationary sources that is required to be maintained by Permit Condition 2.4.

Table 2 - ICE Source Changes

\begin{tabular}{|c|c|c|c|c|l|}
\hline Facility & Engine ID & Action & $\begin{array}{c}\text { Installation } \\
\text { Date }\end{array}$ & $\begin{array}{c}\text { Removal } \\
\text { Date }\end{array}$ & \multicolumn{1}{|c|}{ Purpose } \\
\hline CFA & B8-601 & Installed & 2016 & NA & Replacement for B8-601 included in permit application. \\
\hline CFA & B27-601 & Installed & 2016 & NA & Emergency engine for new project. \\
\hline CFA & ARA-632 & Installed & 2016 & NA & Emergency engine for new project. \\
\hline CFA & CFA-609-002 & Installed & 2016 & NA & Emergency engine for new project. \\
\hline CFA & TAN-601 & Installed & 2017 & NA & Replacement for TAN-601 included in permit application. \\
\hline CFA & B27-607/609 & Installed & 2018 & NA & Emergency engine for new project. \\
\hline RWMC & S-GEN-RE901 & Installed & 2017 & NA & Emergency engine for new project. \\
\hline MFC & ANL-1740 & Installed & 2015 & NA & Emergency engine for new project. \\
\hline MFC & ANL-1729 & Installed & 2017 & NA & Emergency engine for new project. \\
\hline AMWTP & BGEN-RCE-001 & NA & 2014 & NA & Omitted from initial permit application. \\
\hline RWMC & HV-GEN-RE301 & NA & 2007 & NA & Omitted from initial permit application. \\
\hline RWMC & S-GEN-RE401 & NA & 2008 & NA & Omitted from initial permit application. \\
\hline
\end{tabular}


Table 2 (cont.)

\begin{tabular}{|c|c|c|c|c|l|}
\hline Facility & Engine ID & Action & $\begin{array}{c}\text { Installation } \\
\text { Date }\end{array}$ & $\begin{array}{c}\text { Removal } \\
\text { Date }\end{array}$ & \multicolumn{1}{|c|}{ Purpose } \\
\hline RWMC & S-GEN-RE701 & NA & 2011 & NA & Omitted from initial permit application. \\
\hline RWMC & S-GEN-RE801 & NA & 2010 & NA & Omitted from initial permit application. \\
\hline CFA & B8-601 & Removed & NA & 2016 & Replaced by new B8-601 \\
\hline CFA & TAN-601 & Removed & NA & 2017 & Replaced by new TAN-601 \\
\hline
\end{tabular}

NRF removed two boilers from service and installed a new replacement boiler that meets the 40 CFR Part 60 Subpart Dc (Table 3).

\section{Table 3 - Boiler Source Changes}

\begin{tabular}{|c|c|c|c|c|l|}
\hline Facility & Engine ID & Action & $\begin{array}{c}\text { Installation } \\
\text { Date }\end{array}$ & $\begin{array}{c}\text { Removal } \\
\text { Date }\end{array}$ & \multicolumn{1}{|c|}{ Purpose } \\
\hline NRF & $\begin{array}{c}\text { NRF-620-014 } \\
\text { (Boiler No. 1) }\end{array}$ & Removed & 1961 & 2018 & End of useful life. \\
\hline NRF & $\begin{array}{c}\text { NRF-620-012 } \\
\text { (Boiler No. 3) }\end{array}$ & Removed & 1961 & 2018 & End of useful life. \\
\hline NRF & Boiler No. 5 & Installed & 2017 & NA & $\begin{array}{l}\text { Replacement boiler for emission units NRF-620-012 and } \\
\text { NRF-620-014. }\end{array}$ \\
\hline
\end{tabular}

Appendix C contains lists of stationary sources with changes annotated. These changes took place after the initial permit application and are maintained to comply with Permit Condition 2.4. 


\section{Appendix A}

\section{Emissions}


INTENTIONALLY BLANK 
Table A-1; 2018 Monthly Emissions

\begin{tabular}{|c|c|c|c|c|c|c|c|c|c|c|c|c|}
\hline Hazardous Air Pollutants (Tons) & Jan-18 & Feb-18 & Mar-18 & Apr-18 & May-18 & Jun-18 & Jul-18 & Aug-18 & Sep-18 & Oct-18 & Nov-18 & Dec-18 \\
\hline 1,1,1-Trichloroethane & $4.78 \mathrm{E}-04$ & $4.76 \mathrm{E}-04$ & $4.75 \mathrm{E}-04$ & $4.68 \mathrm{E}-04$ & $4.62 \mathrm{E}-04$ & $4.60 \mathrm{E}-04$ & $4.61 \mathrm{E}-04$ & $4.60 \mathrm{E}-04$ & $4.59 \mathrm{E}-04$ & $4.69 \mathrm{E}-04$ & $4.76 \mathrm{E}-04$ & $4.81 \mathrm{E}-04$ \\
\hline 1,1,2,2-Tetrachloroethane & $1.46 \mathrm{E}-05$ & $1.45 \mathrm{E}-05$ & $1.45 \mathrm{E}-05$ & $1.45 \mathrm{E}-05$ & $1.45 \mathrm{E}-05$ & $1.45 \mathrm{E}-05$ & $1.45 \mathrm{E}-05$ & $1.45 \mathrm{E}-05$ & $1.45 \mathrm{E}-05$ & $1.45 \mathrm{E}-05$ & $1.44 \mathrm{E}-05$ & $1.44 \mathrm{E}-05$ \\
\hline 1,1,2-Trichloroethane & $2.01 \mathrm{E}-05$ & $2.00 \mathrm{E}-05$ & $2.00 \mathrm{E}-05$ & $2.01 \mathrm{E}-05$ & $2.00 \mathrm{E}-05$ & $2.00 \mathrm{E}-05$ & $2.00 \mathrm{E}-05$ & $2.00 \mathrm{E}-05$ & $2.00 \mathrm{E}-05$ & $2.00 \mathrm{E}-05$ & $2.00 \mathrm{E}-05$ & $2.00 \mathrm{E}-05$ \\
\hline 1,1-Dichloroethane & $2.44 \mathrm{E}-05$ & $2.44 \mathrm{E}-05$ & $2.44 \mathrm{E}-05$ & $2.44 \mathrm{E}-05$ & $2.44 \mathrm{E}-05$ & $2.44 \mathrm{E}-05$ & $2.44 \mathrm{E}-05$ & $2.44 \mathrm{E}-05$ & $2.44 \mathrm{E}-05$ & $2.44 \mathrm{E}-05$ & $2.44 \mathrm{E}-05$ & $2.44 \mathrm{E}-05$ \\
\hline 1,1-Dichloroethylene & $1.84 \mathrm{E}-05$ & 1.67E-05 & 1.67E-05 & 1.67E-05 & 1.67E-05 & 1.67E-05 & 1.67E-05 & $1.67 \mathrm{E}-05$ & 1.67E-05 & 1.67E-05 & 1.67E-05 & 1.67E-05 \\
\hline 1,2,4-Trichlorobenzene & $N A^{1}$ & NA & NA & NA & NA & NA & NA & NA & NA & NA & NA & NA \\
\hline 1,2-Dibromo-3-chloropropane & NA & NA & NA & NA & NA & NA & NA & NA & NA & NA & NA & NA \\
\hline 1,2-Dichloroethane & $4.86 \mathrm{E}-05$ & $4.86 \mathrm{E}-05$ & $4.86 \mathrm{E}-05$ & $4.86 \mathrm{E}-05$ & $4.86 \mathrm{E}-05$ & $4.86 \mathrm{E}-05$ & $4.86 \mathrm{E}-05$ & $4.86 \mathrm{E}-05$ & $4.86 \mathrm{E}-05$ & $4.86 \mathrm{E}-05$ & $4.86 \mathrm{E}-05$ & $4.86 \mathrm{E}-05$ \\
\hline 1,2-Dichloropropane & $2.38 \mathrm{E}-05$ & $2.37 \mathrm{E}-05$ & 2.37E-05 & $2.38 \mathrm{E}-05$ & 2.37E-05 & $2.37 \mathrm{E}-05$ & 2.37E-05 & 2.37E-05 & 2.37E-05 & 2.37E-05 & $2.37 \mathrm{E}-05$ & 2.37E-05 \\
\hline 1,2-Diphenylhydrazine & NA & NA & NA & NA & NA & NA & NA & NA & NA & NA & NA & NA \\
\hline 1,3-Butadiene & $2.10 \mathrm{E}-05$ & $1.99 \mathrm{E}-05$ & $1.81 \mathrm{E}-05$ & $1.85 \mathrm{E}-05$ & 3.07E-05 & $2.08 \mathrm{E}-05$ & $1.76 \mathrm{E}-05$ & $2.29 \mathrm{E}-05$ & $1.84 \mathrm{E}-05$ & $1.83 \mathrm{E}-05$ & $1.72 \mathrm{E}-05$ & $1.64 \mathrm{E}-05$ \\
\hline 1,3-Dichloropropene & $8.80 \mathrm{E}-08$ & $3.51 \mathrm{E}-08$ & $2.92 \mathrm{E}-08$ & $5.74 \mathrm{E}-08$ & $3.46 \mathrm{E}-08$ & $2.52 \mathrm{E}-08$ & $2.57 \mathrm{E}-08$ & $2.57 \mathrm{E}-08$ & $4.09 \mathrm{E}-08$ & $3.64 \mathrm{E}-08$ & $1.16 \mathrm{E}-08$ & $2.91 \mathrm{E}-09$ \\
\hline 1,4-Dichlorobenzene & $2.65 \mathrm{E}-05$ & $2.65 \mathrm{E}-05$ & $2.65 \mathrm{E}-05$ & $2.65 \mathrm{E}-05$ & $2.65 \mathrm{E}-05$ & $2.65 \mathrm{E}-05$ & $2.65 \mathrm{E}-05$ & $2.65 \mathrm{E}-05$ & $2.65 \mathrm{E}-05$ & $2.65 \mathrm{E}-05$ & $2.65 \mathrm{E}-05$ & $2.65 \mathrm{E}-05$ \\
\hline 1,4 Dioxane & $2.09 \mathrm{E}-06$ & $2.09 \mathrm{E}-06$ & $2.09 \mathrm{E}-06$ & $2.09 \mathrm{E}-06$ & $2.09 \mathrm{E}-06$ & $2.09 \mathrm{E}-06$ & $2.09 \mathrm{E}-06$ & $2.09 \mathrm{E}-06$ & $2.09 \mathrm{E}-06$ & $2.09 \mathrm{E}-06$ & $2.09 \mathrm{E}-06$ & $2.09 \mathrm{E}-06$ \\
\hline 2,2,4-Trimethyl pentane & $2.37 \mathrm{E}-05$ & $2.37 \mathrm{E}-05$ & $2.37 \mathrm{E}-05$ & 2.37E-05 & $2.37 \mathrm{E}-05$ & $2.37 \mathrm{E}-05$ & 2.37E-05 & $2.37 \mathrm{E}-05$ & $2.37 \mathrm{E}-05$ & $2.37 \mathrm{E}-05$ & $2.37 \mathrm{E}-05$ & 2.37E-05 \\
\hline 2,4,5-Trichlorophenol & NA & NA & NA & NA & NA & NA & NA & NA & NA & NA & NA & NA \\
\hline 2,4,6-Trichlorophenol & NA & NA & NA & NA & NA & NA & NA & NA & NA & NA & NA & NA \\
\hline 2,4-Dinitrophenol & NA & NA & NA & NA & NA & NA & NA & NA & NA & NA & NA & NA \\
\hline 2,4-Dinitrotoluene & $6.93 \mathrm{E}-05$ & $6.93 \mathrm{E}-05$ & $6.93 \mathrm{E}-05$ & $6.93 \mathrm{E}-05$ & $6.93 \mathrm{E}-05$ & $6.93 \mathrm{E}-05$ & $6.93 \mathrm{E}-05$ & $6.93 \mathrm{E}-05$ & $6.93 \mathrm{E}-05$ & $6.93 \mathrm{E}-05$ & $6.93 \mathrm{E}-05$ & $6.93 \mathrm{E}-05$ \\
\hline 4-Nitrophenol & NA & NA & NA & NA & NA & NA & NA & NA & NA & NA & NA & NA \\
\hline Acetaldehyde & $4.20 \mathrm{E}-04$ & $3.64 \mathrm{E}-04$ & $3.34 \mathrm{E}-04$ & $3.19 \mathrm{E}-04$ & $5.79 \mathrm{E}-04$ & $3.89 \mathrm{E}-04$ & $3.26 \mathrm{E}-04$ & $4.31 \mathrm{E}-04$ & $3.29 \mathrm{E}-04$ & $3.32 \mathrm{E}-04$ & $3.29 \mathrm{E}-04$ & $3.42 \mathrm{E}-04$ \\
\hline Acetophenone & NA & NA & NA & NA & NA & NA & NA & NA & NA & NA & NA & NA \\
\hline Acrolein & $2.38 \mathrm{E}-04$ & $2.07 \mathrm{E}-04$ & $2.02 \mathrm{E}-04$ & $2.06 \mathrm{E}-04$ & $2.34 \mathrm{E}-04$ & $2.08 \mathrm{E}-04$ & $2.01 \mathrm{E}-04$ & $2.14 \mathrm{E}-04$ & $2.04 \mathrm{E}-04$ & $2.04 \mathrm{E}-04$ & $1.99 \mathrm{E}-04$ & $2.03 \mathrm{E}-04$ \\
\hline Acrylonitrile & NA & NA & NA & NA & NA & NA & NA & NA & NA & NA & NA & NA \\
\hline Aniline & NA & NA & NA & NA & NA & NA & NA & NA & NA & NA & NA & NA \\
\hline Antimony & 1.57E-09 & $1.57 \mathrm{E}-09$ & 1.57E-09 & 1.57E-09 & 1.57E-09 & 1.57E-09 & 1.57E-09 & 1.57E-09 & $1.57 \mathrm{E}-09$ & 1.57E-09 & 1.57E-09 & 1.57E-09 \\
\hline Arsenic & $1.06 \mathrm{E}-04$ & $1.02 \mathrm{E}-04$ & $1.00 \mathrm{E}-04$ & $8.43 \mathrm{E}-05$ & $7.05 \mathrm{E}-05$ & $6.60 \mathrm{E}-05$ & $6.62 \mathrm{E}-05$ & $6.50 \mathrm{E}-05$ & $6.30 \mathrm{E}-05$ & $8.64 \mathrm{E}-05$ & $1.02 \mathrm{E}-04$ & $1.13 \mathrm{E}-04$ \\
\hline Benzene & $2.90 \mathrm{E}-03$ & $5.90 \mathrm{E}-04$ & $5.25 \mathrm{E}-04$ & $5.41 \mathrm{E}-04$ & $9.32 \mathrm{E}-04$ & $5.75 \mathrm{E}-04$ & 4.99E-04 & $6.77 \mathrm{E}-04$ & $5.13 \mathrm{E}-04$ & $5.30 \mathrm{E}-04$ & $5.39 \mathrm{E}-04$ & $1.19 \mathrm{E}-03$ \\
\hline Benzidine & NA & NA & NA & NA & NA & NA & NA & NA & NA & NA & NA & NA \\
\hline Beryllium & $1.05 \mathrm{E}-04$ & $1.01 \mathrm{E}-04$ & $1.00 \mathrm{E}-04$ & $8.84 \mathrm{E}-05$ & $7.81 \mathrm{E}-05$ & $7.47 \mathrm{E}-05$ & $7.49 \mathrm{E}-05$ & $7.40 \mathrm{E}-05$ & $7.25 \mathrm{E}-05$ & $9.00 \mathrm{E}-05$ & $1.02 \mathrm{E}-04$ & $1.10 \mathrm{E}-04$ \\
\hline bis(2-Chloroethyl)ether & NA & NA & NA & NA & NA & NA & NA & NA & NA & NA & NA & NA \\
\hline bis(2-Ethylhexyl)phthalate & $1.41 \mathrm{E}-08$ & $1.41 \mathrm{E}-08$ & $1.41 \mathrm{E}-08$ & $1.41 \mathrm{E}-08$ & $1.41 \mathrm{E}-08$ & $1.41 \mathrm{E}-08$ & $1.41 \mathrm{E}-08$ & $1.41 \mathrm{E}-08$ & $1.41 \mathrm{E}-08$ & $1.41 \mathrm{E}-08$ & $1.41 \mathrm{E}-08$ & $1.41 \mathrm{E}-08$ \\
\hline Bromoform & $5.70 \mathrm{E}-08$ & $5.70 \mathrm{E}-08$ & $5.70 \mathrm{E}-08$ & $5.70 \mathrm{E}-08$ & $5.70 \mathrm{E}-08$ & $5.70 \mathrm{E}-08$ & $5.70 \mathrm{E}-08$ & $5.70 \mathrm{E}-08$ & $5.70 \mathrm{E}-08$ & $5.70 \mathrm{E}-08$ & $5.70 \mathrm{E}-08$ & $5.70 \mathrm{E}-08$ \\
\hline Bromomethane & NA & NA & NA & NA & NA & NA & NA & NA & NA & NA & NA & NA \\
\hline Cadmium & $4.38 \mathrm{E}-04$ & $4.34 \mathrm{E}-04$ & $4.33 \mathrm{E}-04$ & $4.21 \mathrm{E}-04$ & $4.11 \mathrm{E}-04$ & $4.08 \mathrm{E}-04$ & $4.08 \mathrm{E}-04$ & 4.07E-04 & $4.05 \mathrm{E}-04$ & $4.23 \mathrm{E}-04$ & $4.35 \mathrm{E}-04$ & $4.43 \mathrm{E}-04$ \\
\hline Carbon disulfide & $3.80 \mathrm{E}-05$ & $3.80 \mathrm{E}-05$ & $3.80 \mathrm{E}-05$ & $3.80 \mathrm{E}-05$ & $3.80 \mathrm{E}-05$ & $3.80 \mathrm{E}-05$ & $3.80 \mathrm{E}-05$ & $3.80 \mathrm{E}-05$ & $3.80 \mathrm{E}-05$ & $3.80 \mathrm{E}-05$ & $3.80 \mathrm{E}-05$ & $3.80 \mathrm{E}-05$ \\
\hline Carbon Tetrachloride & $1.46 \mathrm{E}-03$ & $1.46 \mathrm{E}-03$ & $1.46 \mathrm{E}-03$ & $1.46 \mathrm{E}-03$ & $1.46 \mathrm{E}-03$ & $1.46 \mathrm{E}-03$ & $1.46 \mathrm{E}-03$ & $1.46 \mathrm{E}-03$ & $1.46 \mathrm{E}-03$ & $1.46 \mathrm{E}-03$ & $1.46 \mathrm{E}-03$ & $1.46 \mathrm{E}-03$ \\
\hline
\end{tabular}


Table A-1 (cont.)

\begin{tabular}{|c|c|c|c|c|c|c|c|c|c|c|c|c|}
\hline Hazardous Air Pollutants (Tons) & Jan-18 & Feb-18 & Mar-18 & Apr-18 & May-18 & Jun-18 & Jul-18 & Aug-18 & Sep-18 & Oct-18 & Nov-18 & Dec-18 \\
\hline Carbonyl sulfide & NA & NA & NA & NA & NA & NA & NA & NA & NA & NA & NA & NA \\
\hline Chlorobenzene & $6.98 \mathrm{E}-05$ & $6.97 \mathrm{E}-05$ & $6.97 \mathrm{E}-05$ & $6.98 \mathrm{E}-05$ & $6.97 \mathrm{E}-05$ & $6.97 \mathrm{E}-05$ & $6.97 \mathrm{E}-05$ & $6.97 \mathrm{E}-05$ & $6.97 \mathrm{E}-05$ & $6.97 \mathrm{E}-05$ & $6.97 \mathrm{E}-05$ & 6.97E-05 \\
\hline Chloroethane (ethyl chloride) & NA & NA & NA & NA & NA & NA & NA & NA & NA & NA & NA & NA \\
\hline Chloroform & $1.33 \mathrm{E}-04$ & $1.33 \mathrm{E}-04$ & $1.33 \mathrm{E}-04$ & $1.33 \mathrm{E}-04$ & $1.33 \mathrm{E}-04$ & $1.33 \mathrm{E}-04$ & $1.33 \mathrm{E}-04$ & $1.33 \mathrm{E}-04$ & $1.33 \mathrm{E}-04$ & $1.33 \mathrm{E}-04$ & $1.33 \mathrm{E}-04$ & $1.33 \mathrm{E}-04$ \\
\hline Chloromethane & NA & NA & NA & NA & NA & NA & NA & NA & NA & NA & NA & NA \\
\hline Chromium & $5.19 \mathrm{E}-05$ & 4.87E-05 & $4.77 \mathrm{E}-05$ & $3.57 \mathrm{E}-05$ & $2.54 \mathrm{E}-05$ & $2.20 \mathrm{E}-05$ & $2.22 \mathrm{E}-05$ & $2.13 \mathrm{E}-05$ & $1.98 \mathrm{E}-05$ & $3.73 \mathrm{E}-05$ & $4.92 \mathrm{E}-05$ & $5.73 \mathrm{E}-05$ \\
\hline Cobalt & NA & NA & NA & NA & NA & NA & NA & NA & NA & NA & NA & NA \\
\hline Cresols (m, p \& o) & $6.17 \mathrm{E}-05$ & $6.17 \mathrm{E}-05$ & $6.17 \mathrm{E}-05$ & $6.17 \mathrm{E}-05$ & $6.17 \mathrm{E}-05$ & $6.17 \mathrm{E}-05$ & $6.17 \mathrm{E}-05$ & $6.17 \mathrm{E}-05$ & $6.17 \mathrm{E}-05$ & $6.17 \mathrm{E}-05$ & $6.17 \mathrm{E}-05$ & $6.17 \mathrm{E}-05$ \\
\hline Dibenzofuran & NA & NA & NA & NA & NA & NA & NA & NA & NA & NA & NA & NA \\
\hline Dimethyl phthalate & NA & NA & NA & NA & NA & NA & NA & NA & NA & NA & NA & NA \\
\hline Ethyl Benzene & $4.94 \mathrm{E}-05$ & $4.87 \mathrm{E}-05$ & $4.86 \mathrm{E}-05$ & $4.68 \mathrm{E}-05$ & $4.51 \mathrm{E}-05$ & $4.46 \mathrm{E}-05$ & $4.46 \mathrm{E}-05$ & $4.45 \mathrm{E}-05$ & $4.43 \mathrm{E}-05$ & $4.70 \mathrm{E}-05$ & $4.88 \mathrm{E}-05$ & $6.45 \mathrm{E}-04$ \\
\hline Ethylene Dibromide & $1.48 \mathrm{E}-07$ & $5.89 \mathrm{E}-08$ & 4.89E-08 & $9.62 \mathrm{E}-08$ & $5.80 \mathrm{E}-08$ & $4.23 \mathrm{E}-08$ & $4.31 \mathrm{E}-08$ & $4.31 \mathrm{E}-08$ & $6.86 \mathrm{E}-08$ & $6.11 \mathrm{E}-08$ & $1.94 \mathrm{E}-08$ & $4.88 \mathrm{E}-09$ \\
\hline Formaldehyde & $5.06 \mathrm{E}-03$ & $4.40 \mathrm{E}-03$ & $4.23 \mathrm{E}-03$ & $2.84 \mathrm{E}-03$ & $2.00 \mathrm{E}-03$ & 1.29E-03 & $1.22 \mathrm{E}-03$ & $1.28 \mathrm{E}-03$ & $9.60 \mathrm{E}-04$ & $3.02 \mathrm{E}-03$ & $4.38 \mathrm{E}-03$ & $5.38 \mathrm{E}-03$ \\
\hline Hexachlorobenzene & $1.84 \mathrm{E}-07$ & $1.84 \mathrm{E}-07$ & $1.84 \mathrm{E}-07$ & $1.84 \mathrm{E}-07$ & $1.84 \mathrm{E}-07$ & $1.84 \mathrm{E}-07$ & $1.84 \mathrm{E}-07$ & $1.84 \mathrm{E}-07$ & $1.84 \mathrm{E}-07$ & $1.84 \mathrm{E}-07$ & $1.84 \mathrm{E}-07$ & $1.84 \mathrm{E}-07$ \\
\hline Hexachlorobutadiene & $7.00 \mathrm{E}-07$ & $7.00 \mathrm{E}-07$ & $7.00 \mathrm{E}-07$ & $7.00 \mathrm{E}-07$ & $7.00 \mathrm{E}-07$ & $7.00 \mathrm{E}-07$ & $7.00 \mathrm{E}-07$ & $7.00 \mathrm{E}-07$ & $7.00 \mathrm{E}-07$ & $7.00 \mathrm{E}-07$ & $7.00 \mathrm{E}-07$ & $7.00 \mathrm{E}-07$ \\
\hline Hexachlorocyclopentadiene & NA & NA & NA & NA & NA & NA & NA & NA & NA & NA & NA & NA \\
\hline Hydrochloric Acid & 1.04E-01 & $1.67 \mathrm{E}-01$ & $1.18 \mathrm{E}-01$ & $1.45 \mathrm{E}-01$ & $1.26 \mathrm{E}-01$ & $1.64 \mathrm{E}-01$ & $1.66 \mathrm{E}-01$ & $6.70 \mathrm{E}-02$ & $3.50 \mathrm{E}-02$ & $5.40 \mathrm{E}-02$ & $5.40 \mathrm{E}-02$ & $5.85 \mathrm{E}-02$ \\
\hline Isophorone & NA & NA & NA & NA & NA & NA & NA & NA & NA & NA & NA & NA \\
\hline Lead & $1.81 \mathrm{E}-03$ & $1.80 \mathrm{E}-03$ & $1.80 \mathrm{E}-03$ & $1.76 \mathrm{E}-03$ & $1.73 \mathrm{E}-03$ & $1.72 \mathrm{E}-03$ & $1.72 \mathrm{E}-03$ & $1.72 \mathrm{E}-03$ & $1.72 \mathrm{E}-03$ & $1.77 \mathrm{E}-03$ & $1.80 \mathrm{E}-03$ & $1.83 \mathrm{E}-03$ \\
\hline Manganese & $7.11 \mathrm{E}-05$ & $6.46 \mathrm{E}-05$ & $6.26 \mathrm{E}-05$ & 3.87E-05 & $1.80 \mathrm{E}-05$ & $1.12 \mathrm{E}-05$ & $1.16 \mathrm{E}-05$ & $9.78 \mathrm{E}-06$ & $6.78 \mathrm{E}-06$ & 4.19E-05 & $6.57 \mathrm{E}-05$ & $8.19 \mathrm{E}-05$ \\
\hline Mercury & $7.54 \mathrm{E}-05$ & $2.01 \mathrm{E}-04$ & $7.12 \mathrm{E}-05$ & $5.92 \mathrm{E}-05$ & $4.88 \mathrm{E}-05$ & $4.54 \mathrm{E}-05$ & $4.56 \mathrm{E}-05$ & 4.47E-05 & $4.32 \mathrm{E}-05$ & $6.08 \mathrm{E}-05$ & $7.27 \mathrm{E}-05$ & $8.08 \mathrm{E}-05$ \\
\hline Methanol & $1.89 \mathrm{E}-04$ & $1.76 \mathrm{E}-04$ & $1.75 \mathrm{E}-04$ & $1.81 \mathrm{E}-04$ & $1.76 \mathrm{E}-04$ & $1.74 \mathrm{E}-04$ & $1.74 \mathrm{E}-04$ & $1.74 \mathrm{E}-04$ & $1.77 \mathrm{E}-04$ & $1.76 \mathrm{E}-04$ & 1.70E-04 & $2.02 \mathrm{E}-04$ \\
\hline Methyl isobutyl ketone & $4.38 \mathrm{E}-06$ & $4.38 \mathrm{E}-06$ & $4.38 \mathrm{E}-06$ & $4.38 \mathrm{E}-06$ & $4.38 \mathrm{E}-06$ & $4.38 \mathrm{E}-06$ & $4.38 \mathrm{E}-06$ & $4.38 \mathrm{E}-06$ & $4.38 \mathrm{E}-06$ & $4.38 \mathrm{E}-06$ & $4.38 \mathrm{E}-06$ & $4.22 \mathrm{E}-05$ \\
\hline Methylene chloride & $2.26 \mathrm{E}-04$ & $2.26 \mathrm{E}-04$ & $2.26 \mathrm{E}-04$ & $2.26 \mathrm{E}-04$ & $2.26 \mathrm{E}-04$ & $2.26 \mathrm{E}-04$ & $2.26 \mathrm{E}-04$ & $2.26 \mathrm{E}-04$ & $2.26 \mathrm{E}-04$ & $2.26 \mathrm{E}-04$ & $2.26 \mathrm{E}-04$ & $2.26 \mathrm{E}-04$ \\
\hline Naphthalene & $5.13 \mathrm{E}-04$ & $1.14 \mathrm{E}-04$ & $1.04 \mathrm{E}-04$ & $7.58 \mathrm{E}-05$ & $8.95 \mathrm{E}-05$ & $3.78 \mathrm{E}-05$ & $3.15 \mathrm{E}-05$ & $4.93 \mathrm{E}-05$ & $2.72 \mathrm{E}-05$ & $7.68 \mathrm{E}-05$ & $1.10 \mathrm{E}-04$ & $2.78 \mathrm{E}-04$ \\
\hline Nickel & $3.68 \mathrm{E}-04$ & 3.64E-04 & $3.63 \mathrm{E}-04$ & $3.51 \mathrm{E}-04$ & $3.41 \mathrm{E}-04$ & $3.38 \mathrm{E}-04$ & $3.38 \mathrm{E}-04$ & 3.37E-04 & $3.35 \mathrm{E}-04$ & $3.53 \mathrm{E}-04$ & $3.65 \mathrm{E}-04$ & $3.73 \mathrm{E}-04$ \\
\hline Nitrobenzene & $7.68 \mathrm{E}-05$ & $7.68 \mathrm{E}-05$ & $7.68 \mathrm{E}-05$ & $7.68 \mathrm{E}-05$ & $7.68 \mathrm{E}-05$ & $7.68 \mathrm{E}-05$ & $7.68 \mathrm{E}-05$ & $7.68 \mathrm{E}-05$ & $7.68 \mathrm{E}-05$ & $7.68 \mathrm{E}-05$ & $7.68 \mathrm{E}-05$ & $7.68 \mathrm{E}-05$ \\
\hline $\mathrm{N}$-Nitrosodimethylamine & NA & NA & NA & NA & NA & NA & NA & NA & NA & NA & NA & NA \\
\hline Polychlorinated biphenyls & $3.13 \mathrm{E}-03$ & $3.13 \mathrm{E}-03$ & $3.13 \mathrm{E}-03$ & $3.13 \mathrm{E}-03$ & $3.13 \mathrm{E}-03$ & $3.13 \mathrm{E}-03$ & $3.13 \mathrm{E}-03$ & $3.13 \mathrm{E}-03$ & $3.13 \mathrm{E}-03$ & $3.13 \mathrm{E}-03$ & $3.13 \mathrm{E}-03$ & $3.13 \mathrm{E}-03$ \\
\hline Pentachloronitrobenzene & NA & NA & NA & NA & NA & NA & NA & NA & NA & NA & NA & NA \\
\hline Pentachlorophenol & $3.80 \mathrm{E}-04$ & $3.80 \mathrm{E}-04$ & $3.80 \mathrm{E}-04$ & $3.80 \mathrm{E}-04$ & $3.80 \mathrm{E}-04$ & $3.80 \mathrm{E}-04$ & $3.80 \mathrm{E}-04$ & $3.80 \mathrm{E}-04$ & $3.80 \mathrm{E}-04$ & $3.80 \mathrm{E}-04$ & $3.80 \mathrm{E}-04$ & $3.80 \mathrm{E}-04$ \\
\hline Phenol & NA & NA & NA & NA & NA & NA & NA & NA & NA & NA & NA & NA \\
\hline Phosphorus & NA & NA & NA & NA & NA & NA & NA & NA & NA & NA & NA & NA \\
\hline Styrene & $8.25 \mathrm{E}-08$ & $3.29 \mathrm{E}-08$ & $2.73 \mathrm{E}-08$ & $5.38 \mathrm{E}-08$ & $3.24 \mathrm{E}-08$ & $2.37 \mathrm{E}-08$ & $2.41 \mathrm{E}-08$ & $2.41 \mathrm{E}-08$ & $3.83 \mathrm{E}-08$ & $3.41 \mathrm{E}-08$ & $1.08 \mathrm{E}-08$ & $2.73 \mathrm{E}-09$ \\
\hline Tetrachloroethylene & $5.88 \mathrm{E}-05$ & $5.88 \mathrm{E}-05$ & $5.88 \mathrm{E}-05$ & $5.88 \mathrm{E}-05$ & $5.88 \mathrm{E}-05$ & $5.88 \mathrm{E}-05$ & $5.88 \mathrm{E}-05$ & $5.88 \mathrm{E}-05$ & $5.88 \mathrm{E}-05$ & $5.88 \mathrm{E}-05$ & $5.88 \mathrm{E}-05$ & $5.88 \mathrm{E}-05$ \\
\hline Toluene & $5.37 \mathrm{E}-03$ & $4.48 \mathrm{E}-03$ & 4.44E-03 & 4.27E-03 & $4.28 \mathrm{E}-03$ & $4.08 \mathrm{E}-03$ & 4.05E-03 & $4.11 \mathrm{E}-03$ & $4.02 \mathrm{E}-03$ & 4.29E-03 & 4.47E-03 & 5.37E-03 \\
\hline
\end{tabular}


Table A-1 (cont.)

\begin{tabular}{|c|c|c|c|c|c|c|c|c|c|c|c|c|}
\hline Hazardous Air Pollutants (Tons) & Jan-18 & Feb-18 & Mar-18 & Apr-18 & May-18 & Jun-18 & Jul-18 & Aug-18 & Sep-18 & Oct-18 & Nov-18 & Dec-18 \\
\hline Trichloroethylene & $1.08 \mathrm{E}-04$ & $1.08 \mathrm{E}-04$ & $1.08 \mathrm{E}-04$ & $1.08 \mathrm{E}-04$ & $1.08 \mathrm{E}-04$ & $1.08 \mathrm{E}-04$ & $1.08 \mathrm{E}-04$ & $1.08 \mathrm{E}-04$ & $1.08 \mathrm{E}-04$ & $1.08 \mathrm{E}-04$ & $1.08 \mathrm{E}-04$ & $1.08 \mathrm{E}-04$ \\
\hline Vinyl Chloride & $3.31 \mathrm{E}-07$ & $3.01 \mathrm{E}-07$ & 2.97E-07 & $3.13 \mathrm{E}-07$ & $3.01 \mathrm{E}-07$ & $2.95 \mathrm{E}-07$ & $2.96 \mathrm{E}-07$ & $2.96 \mathrm{E}-07$ & $3.04 \mathrm{E}-07$ & $3.02 \mathrm{E}-07$ & $2.88 \mathrm{E}-07$ & $2.83 \mathrm{E}-07$ \\
\hline Xylene & $1.26 \mathrm{E}-03$ & $6.86 \mathrm{E}-04$ & $6.68 \mathrm{E}-04$ & $6.68 \mathrm{E}-04$ & $7.83 \mathrm{E}-04$ & $6.81 \mathrm{E}-04$ & $6.58 \mathrm{E}-04$ & 7.09E-04 & $6.61 \mathrm{E}-04$ & $6.68 \mathrm{E}-04$ & $6.72 \mathrm{E}-04$ & $2.80 \mathrm{E}-03$ \\
\hline Total & $1.30 \mathrm{E}-01$ & $1.88 \mathrm{E}-01$ & $1.38 \mathrm{E}-01$ & $1.64 \mathrm{E}-01$ & $1.44 \mathrm{E}-01$ & $1.81 \mathrm{E}-01$ & $1.83 \mathrm{E}-01$ & $8.43 \mathrm{E}-02$ & $5.14 \mathrm{E}-02$ & $7.33 \mathrm{E}-02$ & $7.51 \mathrm{E}-02$ & $8.56 \mathrm{E}-02$ \\
\hline Maximum Single HAP Emission & $1.04 \mathrm{E}-01$ & $1.67 \mathrm{E}-01$ & $1.18 \mathrm{E}-01$ & $1.45 \mathrm{E}-01$ & $1.26 \mathrm{E}-01$ & $1.64 \mathrm{E}-01$ & $1.66 \mathrm{E}-01$ & $6.70 \mathrm{E}-02$ & $3.50 \mathrm{E}-02$ & $5.40 \mathrm{E}-02$ & $5.40 \mathrm{E}-02$ & $5.85 \mathrm{E}-02$ \\
\hline Maximum Single HAP Emitted & $\begin{array}{l}\text { Hydrochloric } \\
\text { Acid }\end{array}$ & $\begin{array}{l}\text { Hydrochloric } \\
\text { Acid }\end{array}$ & $\begin{array}{l}\text { Hydrochloric } \\
\text { Acid }\end{array}$ & $\begin{array}{l}\text { Hydrochloric } \\
\text { Acid }\end{array}$ & $\begin{array}{l}\text { Hydrochloric } \\
\text { Acid }\end{array}$ & $\begin{array}{l}\text { Hydrochloric } \\
\text { Acid }\end{array}$ & $\begin{array}{l}\text { Hydrochloric } \\
\text { Acid }\end{array}$ & $\begin{array}{l}\text { Hydrochloric } \\
\text { Acid }\end{array}$ & $\begin{array}{c}\text { Hydrochloric } \\
\text { Acid }\end{array}$ & $\begin{array}{c}\text { Hydrochloric } \\
\text { Acid }\end{array}$ & $\begin{array}{l}\text { Hydrochloric } \\
\text { Acid }\end{array}$ & $\begin{array}{l}\text { Hydrochloric } \\
\text { Acid }\end{array}$ \\
\hline
\end{tabular}

\begin{tabular}{|c|c|c|c|c|c|c|c|c|c|c|c|c|}
\hline Criteria Air Pollutants & Jan-18 & Feb-18 & Mar-18 & Apr-18 & May-18 & Jun-18 & Jul-18 & Aug-18 & Sep-18 & Oct-18 & Nov-18 & Dec-18 \\
\hline $\mathrm{SO} 2$ & $5.70 \mathrm{E}-02$ & $2.14 \mathrm{E}-01$ & $1.99 \mathrm{E}-01$ & $1.96 \mathrm{E}-01$ & $2.74 \mathrm{E}-01$ & $2.01 \mathrm{E}-01$ & $1.76 \mathrm{E}-01$ & $2.15 \mathrm{E}-01$ & $1.76 \mathrm{E}-01$ & $1.90 \mathrm{E}-01$ & 5.07E-02 & 2.07E-01 \\
\hline NOx & $2.62 E+00$ & $3.43 E+00$ & $3.07 E+00$ & $2.77 E+00$ & $3.84 \mathrm{E}+00$ & $2.07 E+00$ & $2.06 \mathrm{E}+00$ & $2.91 \mathrm{E}+00$ & $1.66 \mathrm{E}+00$ & $2.58 \mathrm{E}+00$ & $2.34 \mathrm{E}+00$ & $6.31 \mathrm{E}+00$ \\
\hline $\mathrm{CO}$ & 7.47E-01 & $1.15 \mathrm{E}+00$ & $1.05 \mathrm{E}+00$ & 9.95E-01 & $1.20 \mathrm{E}+00$ & $7.68 \mathrm{E}-01$ & $6.89 \mathrm{E}-01$ & $8.65 \mathrm{E}-01$ & $6.90 \mathrm{E}-01$ & $9.22 \mathrm{E}-01$ & $5.96 \mathrm{E}-01$ & $1.91 \mathrm{E}+00$ \\
\hline PM-10/2.5 \& Condensable & $3.28 \mathrm{E}-01$ & $4.00 \mathrm{E}-01$ & $3.76 \mathrm{E}-01$ & $2.89 \mathrm{E}-01$ & $3.02 \mathrm{E}-01$ & $1.88 \mathrm{E}-01$ & $1.64 \mathrm{E}-01$ & $2.03 \mathrm{E}-01$ & $1.46 \mathrm{E}-01$ & $2.91 \mathrm{E}-01$ & $3.02 \mathrm{E}-01$ & $5.16 \mathrm{E}-01$ \\
\hline VOCs, as VOCs & $6.65 \mathrm{E}-02$ & $2.48 \mathrm{E}-01$ & $2.29 \mathrm{E}-01$ & $2.34 \mathrm{E}-01$ & $3.39 \mathrm{E}-01$ & $2.37 \mathrm{E}-01$ & $2.08 \mathrm{E}-01$ & $2.61 \mathrm{E}-01$ & $2.09 \mathrm{E}-01$ & $2.22 \mathrm{E}-01$ & $6.01 \mathrm{E}-02$ & $3.43 \mathrm{E}-01$ \\
\hline
\end{tabular}

Note 1: NA indicates no emissions for Calendar Year 2018. 
Table A-2; 12 Month Rolling Emissions

\begin{tabular}{|c|c|c|c|c|c|c|c|c|c|c|c|c|}
\hline $\begin{array}{l}\text { Hazardous Air Pollutant } \\
\text { (Tons) }\end{array}$ & Jan-18 & Feb-18 & Mar-18 & Apr-18 & May-18 & Jun-18 & Jul-18 & Aug-18 & Sep-18 & Oct-18 & Nov-18 & Dec-18 \\
\hline 1,1,1-Trichloroethane & $6.31 \mathrm{E}-01$ & $6.02 \mathrm{E}-01$ & $5.73 \mathrm{E}-01$ & $5.10 \mathrm{E}-01$ & 4.47E-01 & $3.84 \mathrm{E}-01$ & $3.21 \mathrm{E}-01$ & $2.58 \mathrm{E}-01$ & $1.95 \mathrm{E}-01$ & $1.32 \mathrm{E}-01$ & $6.86 \mathrm{E}-02$ & $5.63 \mathrm{E}-03$ \\
\hline 1,1,2,2-Tetrachloroethane & $1.05 \mathrm{E}-03$ & $1.03 \mathrm{E}-03$ & $1.00 \mathrm{E}-03$ & $9.13 \mathrm{E}-04$ & $8.20 \mathrm{E}-04$ & $7.28 \mathrm{E}-04$ & $6.35 \mathrm{E}-04$ & $5.43 \mathrm{E}-04$ & $4.51 \mathrm{E}-04$ & $3.59 \mathrm{E}-04$ & $2.66 \mathrm{E}-04$ & $1.74 \mathrm{E}-04$ \\
\hline 1,1,2-Trichloroethane & $8.92 \mathrm{E}-04$ & 8.67E-04 & $8.42 \mathrm{E}-04$ & $7.75 \mathrm{E}-04$ & $7.08 \mathrm{E}-04$ & $6.42 \mathrm{E}-04$ & $5.75 \mathrm{E}-04$ & $5.08 \mathrm{E}-04$ & $4.41 \mathrm{E}-04$ & $3.74 \mathrm{E}-04$ & $3.07 \mathrm{E}-04$ & 2.40E-04 \\
\hline 1,1-Dichloroethane & $2.51 \mathrm{E}-05$ & $4.94 \mathrm{E}-05$ & $7.38 \mathrm{E}-05$ & $9.82 \mathrm{E}-05$ & $1.23 \mathrm{E}-04$ & $1.47 \mathrm{E}-04$ & $1.71 \mathrm{E}-04$ & $1.96 \mathrm{E}-04$ & $2.20 \mathrm{E}-04$ & 2.44E-04 & $2.68 \mathrm{E}-04$ & 2.93E-04 \\
\hline 1,1-Dichloroethylene & $3.86 \mathrm{E}-02$ & $3.51 \mathrm{E}-02$ & $3.16 \mathrm{E}-02$ & $2.81 \mathrm{E}-02$ & $2.46 \mathrm{E}-02$ & $2.11 \mathrm{E}-02$ & $1.77 \mathrm{E}-02$ & $1.42 \mathrm{E}-02$ & $1.07 \mathrm{E}-02$ & $7.18 \mathrm{E}-03$ & $3.69 \mathrm{E}-03$ & 2.02E-04 \\
\hline 1,2,4-Trichlorobenzene & $\mathrm{NA}^{1}$ & NA & NA & NA & NA & NA & NA & NA & NA & NA & NA & NA \\
\hline 1,2-Dibromo-3-chloropropane & NA & NA & NA & NA & NA & NA & NA & NA & NA & NA & NA & NA \\
\hline 1,2-Dichloroethane & $2.76 \mathrm{E}-02$ & $2.52 \mathrm{E}-02$ & $2.28 \mathrm{E}-02$ & $2.04 \mathrm{E}-02$ & $1.79 \mathrm{E}-02$ & $1.54 \mathrm{E}-02$ & $1.30 \mathrm{E}-02$ & $1.05 \mathrm{E}-02$ & $8.01 \mathrm{E}-03$ & $5.53 \mathrm{E}-03$ & $3.06 \mathrm{E}-03$ & 5.83E-04 \\
\hline 1,2-Dichloropropane & 4.50E-04 & 4.35E-04 & $4.20 \mathrm{E}-04$ & 4.05E-04 & $3.90 \mathrm{E}-04$ & $3.75 \mathrm{E}-04$ & $3.60 \mathrm{E}-04$ & $3.45 \mathrm{E}-04$ & $3.30 \mathrm{E}-04$ & $3.15 \mathrm{E}-04$ & $3.00 \mathrm{E}-04$ & 2.85E-04 \\
\hline 1,2-Diphenylhydrazine & NA & NA & NA & NA & NA & NA & NA & NA & NA & NA & NA & NA \\
\hline 1,3-Butadiene & $1.37 \mathrm{E}-03$ & $1.33 \mathrm{E}-03$ & $1.28 \mathrm{E}-03$ & $1.17 \mathrm{E}-03$ & $1.07 \mathrm{E}-03$ & 9.50E-04 & $8.31 \mathrm{E}-04$ & 7.17E-04 & $5.99 \mathrm{E}-04$ & 4.80E-04 & $3.62 \mathrm{E}-04$ & 2.40E-04 \\
\hline 1,3-Dichloropropene & $6.52 \mathrm{E}-07$ & $6.69 \mathrm{E}-07$ & $6.72 \mathrm{E}-07$ & $7.10 \mathrm{E}-07$ & 7.19E-07 & $7.22 \mathrm{E}-07$ & $7.08 \mathrm{E}-07$ & $6.95 \mathrm{E}-07$ & $6.48 \mathrm{E}-07$ & $5.71 \mathrm{E}-07$ & 5.27E-07 & 4.13E-07 \\
\hline 1,4-Dichlorobenzene & $3.10 \mathrm{E}-02$ & $2.96 \mathrm{E}-02$ & $2.82 \mathrm{E}-02$ & $2.51 \mathrm{E}-02$ & $2.20 \mathrm{E}-02$ & $1.89 \mathrm{E}-02$ & $1.58 \mathrm{E}-02$ & $1.27 \mathrm{E}-02$ & $9.61 \mathrm{E}-03$ & $6.51 \mathrm{E}-03$ & $3.42 \mathrm{E}-03$ & $3.18 \mathrm{E}-04$ \\
\hline 1,4 Dioxane & $2.51 \mathrm{E}-05$ & $2.51 \mathrm{E}-05$ & $2.51 \mathrm{E}-05$ & $2.51 \mathrm{E}-05$ & $2.51 \mathrm{E}-05$ & $2.51 \mathrm{E}-05$ & $2.51 \mathrm{E}-05$ & $2.51 \mathrm{E}-05$ & $2.51 \mathrm{E}-05$ & $2.51 \mathrm{E}-05$ & $2.51 \mathrm{E}-05$ & $2.51 \mathrm{E}-05$ \\
\hline 2,2,4-Trimethyl pentane & $1.03 \mathrm{E}-03$ & $1.03 \mathrm{E}-03$ & $1.03 \mathrm{E}-03$ & $1.03 \mathrm{E}-03$ & $1.03 \mathrm{E}-03$ & $1.03 \mathrm{E}-03$ & $1.03 \mathrm{E}-03$ & $1.03 \mathrm{E}-03$ & $7.81 \mathrm{E}-04$ & $5.33 \mathrm{E}-04$ & $2.84 \mathrm{E}-04$ & $2.84 \mathrm{E}-04$ \\
\hline 2,4,5-Trichlorophenol & NA & NA & NA & NA & NA & NA & NA & NA & NA & NA & NA & NA \\
\hline 2,4,6-Trichlorophenol & NA & NA & NA & NA & NA & NA & NA & NA & NA & NA & NA & NA \\
\hline 2,4-Dinitrophenol & NA & NA & NA & NA & NA & NA & NA & NA & NA & NA & NA & NA \\
\hline 2,4-Dinitrotoluene & 7.00E-03 & $6.73 \mathrm{E}-03$ & $6.46 \mathrm{E}-03$ & $5.83 \mathrm{E}-03$ & $5.21 \mathrm{E}-03$ & $4.58 \mathrm{E}-03$ & 3.96E-03 & $3.33 \mathrm{E}-03$ & $2.71 \mathrm{E}-03$ & $2.08 \mathrm{E}-03$ & $1.46 \mathrm{E}-03$ & $8.32 \mathrm{E}-04$ \\
\hline 4-Nitrophenol & NA & NA & NA & NA & NA & NA & NA & NA & NA & NA & NA & NA \\
\hline Acetaldehyde & $2.65 \mathrm{E}-02$ & $2.57 \mathrm{E}-02$ & $2.47 \mathrm{E}-02$ & $2.25 \mathrm{E}-02$ & $2.04 \mathrm{E}-02$ & $1.82 \mathrm{E}-02$ & $1.59 \mathrm{E}-02$ & $1.36 \mathrm{E}-02$ & $1.13 \mathrm{E}-02$ & $9.06 \mathrm{E}-03$ & $6.79 \mathrm{E}-03$ & $4.50 \mathrm{E}-03$ \\
\hline Acetophenone & NA & NA & NA & NA & NA & NA & NA & NA & NA & NA & NA & NA \\
\hline Acrolein & 4.27E-03 & $4.25 \mathrm{E}-03$ & $4.23 \mathrm{E}-03$ & 4.06E-03 & $3.90 \mathrm{E}-03$ & $3.71 \mathrm{E}-03$ & $3.52 \mathrm{E}-03$ & $3.33 \mathrm{E}-03$ & $3.13 \mathrm{E}-03$ & $2.93 \mathrm{E}-03$ & $2.73 \mathrm{E}-03$ & $2.52 \mathrm{E}-03$ \\
\hline Acrylonitrile & NA & NA & NA & NA & NA & NA & NA & NA & NA & NA & NA & NA \\
\hline Aniline & NA & NA & NA & NA & NA & NA & NA & NA & NA & NA & NA & NA \\
\hline Antimony & $1.57 \mathrm{E}-09$ & $3.14 \mathrm{E}-09$ & $4.71 \mathrm{E}-09$ & $6.28 \mathrm{E}-09$ & $7.85 \mathrm{E}-09$ & $9.42 \mathrm{E}-09$ & $1.10 \mathrm{E}-08$ & $1.26 \mathrm{E}-08$ & $1.41 \mathrm{E}-08$ & $1.57 \mathrm{E}-08$ & $1.73 \mathrm{E}-08$ & $1.88 \mathrm{E}-08$ \\
\hline Arsenic & $3.25 \mathrm{E}-04$ & $4.02 \mathrm{E}-04$ & $4.76 \mathrm{E}-04$ & $5.38 \mathrm{E}-04$ & $5.95 \mathrm{E}-04$ & $6.56 \mathrm{E}-04$ & 7.17E-04 & 7.77E-04 & $8.36 \mathrm{E}-04$ & $9.00 \mathrm{E}-04$ & $9.62 \mathrm{E}-04$ & $1.02 \mathrm{E}-03$ \\
\hline Benzene & $1.55 \mathrm{E}-01$ & $1.42 \mathrm{E}-01$ & $1.29 \mathrm{E}-01$ & $1.15 \mathrm{E}-01$ & $1.03 \mathrm{E}-01$ & $8.94 \mathrm{E}-02$ & $7.62 \mathrm{E}-02$ & $6.27 \mathrm{E}-02$ & 4.93E-02 & $3.61 \mathrm{E}-02$ & $2.29 \mathrm{E}-02$ & $1.00 \mathrm{E}-02$ \\
\hline Benzidine & NA & NA & NA & NA & NA & NA & NA & NA & NA & NA & NA & NA \\
\hline Beryllium & $2.69 \mathrm{E}-04$ & $3.52 \mathrm{E}-04$ & 4.32E-04 & $5.04 \mathrm{E}-04$ & $5.72 \mathrm{E}-04$ & $6.43 \mathrm{E}-04$ & $7.14 \mathrm{E}-04$ & $7.84 \mathrm{E}-04$ & $8.53 \mathrm{E}-04$ & $9.27 \mathrm{E}-04$ & 9.99E-04 & 1.07E-03 \\
\hline bis(2-Chloroethyl)ether & NA & NA & NA & NA & NA & NA & NA & NA & NA & NA & NA & NA \\
\hline bis(2-Ethylhexyl)phthalate & $1.41 \mathrm{E}-08$ & $2.81 \mathrm{E}-08$ & $4.22 \mathrm{E}-08$ & $5.62 \mathrm{E}-08$ & $7.03 \mathrm{E}-08$ & 8.43E-08 & $9.84 \mathrm{E}-08$ & $1.12 \mathrm{E}-07$ & $1.26 \mathrm{E}-07$ & $1.41 \mathrm{E}-07$ & $1.55 \mathrm{E}-07$ & 1.69E-07 \\
\hline Bromoform & $5.70 \mathrm{E}-08$ & $1.14 \mathrm{E}-07$ & $1.71 \mathrm{E}-07$ & $2.28 \mathrm{E}-07$ & $2.85 \mathrm{E}-07$ & $3.42 \mathrm{E}-07$ & 3.99E-07 & $4.56 \mathrm{E}-07$ & $5.13 \mathrm{E}-07$ & $5.70 \mathrm{E}-07$ & $6.27 \mathrm{E}-07$ & $6.84 \mathrm{E}-07$ \\
\hline Bromomethane & NA & NA & NA & NA & NA & NA & NA & NA & NA & NA & NA & NA \\
\hline Cadmium & $4.27 \mathrm{E}-03$ & $4.35 \mathrm{E}-03$ & $4.43 \mathrm{E}-03$ & 4.50E-03 & 4.57E-03 & 4.64E-03 & $4.71 \mathrm{E}-03$ & $4.78 \mathrm{E}-03$ & 4.85E-03 & $4.92 \mathrm{E}-03$ & 4.99E-03 & 5.07E-03 \\
\hline
\end{tabular}


Table A-2 (cont.)

\begin{tabular}{|c|c|c|c|c|c|c|c|c|c|c|c|c|}
\hline $\begin{array}{l}\text { Hazardous Air Pollutant } \\
\text { (Tons) }\end{array}$ & Jan-18 & Feb-18 & Mar-18 & Apr-18 & May-18 & Jun-18 & Jul-18 & Aug-18 & Sep-18 & Oct-18 & Nov-18 & Dec-18 \\
\hline Carbon disulfide & $3.80 \mathrm{E}-05$ & $7.60 \mathrm{E}-05$ & $1.14 \mathrm{E}-04$ & $1.52 \mathrm{E}-04$ & $1.90 \mathrm{E}-04$ & $2.28 \mathrm{E}-04$ & $2.66 \mathrm{E}-04$ & $3.04 \mathrm{E}-04$ & $3.42 \mathrm{E}-04$ & $3.80 \mathrm{E}-04$ & $4.18 \mathrm{E}-04$ & 4.56E-04 \\
\hline Carbon Tetrachloride & $1.85 \mathrm{E}-01$ & $1.73 \mathrm{E}-01$ & $1.61 \mathrm{E}-01$ & $1.45 \mathrm{E}-01$ & $1.29 \mathrm{E}-01$ & $1.13 \mathrm{E}-01$ & $9.74 \mathrm{E}-02$ & $8.14 \mathrm{E}-02$ & $6.55 \mathrm{E}-02$ & $4.95 \mathrm{E}-02$ & $3.35 \mathrm{E}-02$ & $1.75 \mathrm{E}-02$ \\
\hline Carbonyl sulfide & NA & NA & NA & NA & NA & NA & NA & NA & NA & NA & NA & NA \\
\hline Chlorobenzene & $3.08 \mathrm{E}-02$ & $2.94 \mathrm{E}-02$ & $2.81 \mathrm{E}-02$ & $2.51 \mathrm{E}-02$ & $2.20 \mathrm{E}-02$ & $1.90 \mathrm{E}-02$ & $1.60 \mathrm{E}-02$ & $1.30 \mathrm{E}-02$ & $9.93 \mathrm{E}-03$ & $6.90 \mathrm{E}-03$ & $3.87 \mathrm{E}-03$ & 8.37E-04 \\
\hline Chloroethane & NA & NA & NA & NA & NA & NA & NA & NA & NA & NA & NA & NA \\
\hline Chloroform & $1.90 \mathrm{E}+00$ & $1.73 \mathrm{E}+00$ & $1.56 \mathrm{E}+00$ & $1.39 \mathrm{E}+00$ & $1.21 \mathrm{E}+00$ & $1.04 \mathrm{E}+00$ & $8.68 \mathrm{E}-01$ & $6.95 \mathrm{E}-01$ & $5.21 \mathrm{E}-01$ & $3.48 \mathrm{E}-01$ & $1.75 \mathrm{E}-01$ & $1.60 \mathrm{E}-03$ \\
\hline Chloromethane & NA & NA & NA & NA & NA & NA & NA & NA & NA & NA & NA & NA \\
\hline Chromium & $2.16 \mathrm{E}-04$ & $2.47 \mathrm{E}-04$ & $2.74 \mathrm{E}-04$ & 2.93E-04 & 3.09E-04 & $3.27 \mathrm{E}-04$ & $3.45 \mathrm{E}-04$ & $3.63 \mathrm{E}-04$ & $3.79 \mathrm{E}-04$ & $4.00 \mathrm{E}-04$ & 4.19E-04 & $4.38 \mathrm{E}-04$ \\
\hline Cobalt & NA & NA & NA & NA & NA & NA & NA & NA & NA & NA & NA & NA \\
\hline Cresols (m, p \& o) & $6.17 \mathrm{E}-02$ & $5.89 \mathrm{E}-02$ & $5.62 \mathrm{E}-02$ & $5.00 \mathrm{E}-02$ & $4.38 \mathrm{E}-02$ & $3.77 \mathrm{E}-02$ & $3.15 \mathrm{E}-02$ & $2.54 \mathrm{E}-02$ & $1.92 \mathrm{E}-02$ & $1.31 \mathrm{E}-02$ & $6.90 \mathrm{E}-03$ & $7.40 \mathrm{E}-04$ \\
\hline Cyanide & $6.15 \mathrm{E}-02$ & $5.88 \mathrm{E}-02$ & $5.61 \mathrm{E}-02$ & $5.00 \mathrm{E}-02$ & $4.39 \mathrm{E}-02$ & $3.78 \mathrm{E}-02$ & $3.17 \mathrm{E}-02$ & $2.56 \mathrm{E}-02$ & $1.95 \mathrm{E}-02$ & $1.35 \mathrm{E}-02$ & 7.37E-03 & $1.28 \mathrm{E}-03$ \\
\hline Dibenzofuran & NA & NA & NA & NA & NA & NA & NA & NA & NA & NA & NA & NA \\
\hline Dimethyl phthalate & NA & NA & NA & NA & NA & NA & NA & NA & NA & NA & NA & NA \\
\hline Ethyl Benzene & $3.18 \mathrm{E}-02$ & $3.04 \mathrm{E}-02$ & $2.89 \mathrm{E}-02$ & $2.57 \mathrm{E}-02$ & $2.26 \mathrm{E}-02$ & $1.94 \mathrm{E}-02$ & $1.63 \mathrm{E}-02$ & $1.30 \mathrm{E}-02$ & $9.93 \mathrm{E}-03$ & $6.84 \mathrm{E}-03$ & $3.74 \mathrm{E}-03$ & $1.16 \mathrm{E}-03$ \\
\hline \begin{tabular}{|l|} 
Ethylene Dibromide \\
\end{tabular} & 1.09E-06 & $1.12 \mathrm{E}-06$ & $1.13 \mathrm{E}-06$ & $1.19 \mathrm{E}-06$ & $1.21 \mathrm{E}-06$ & $1.21 \mathrm{E}-06$ & 1.19E-06 & $1.17 \mathrm{E}-06$ & $1.09 \mathrm{E}-06$ & $9.57 \mathrm{E}-07$ & $8.84 \mathrm{E}-07$ & $6.92 \mathrm{E}-07$ \\
\hline Formaldehyde & $2.66 \mathrm{E}-02$ & $2.88 \mathrm{E}-02$ & $3.05 \mathrm{E}-02$ & $3.13 \mathrm{E}-02$ & 3.19E-02 & $3.26 \mathrm{E}-02$ & $3.30 \mathrm{E}-02$ & $3.36 \mathrm{E}-02$ & $3.38 \mathrm{E}-02$ & $3.47 \mathrm{E}-02$ & $3.54 \mathrm{E}-02$ & $3.60 \mathrm{E}-02$ \\
\hline Hexachlorobenzene & $1.65 \mathrm{E}-03$ & $1.57 \mathrm{E}-03$ & 1.49E-03 & $1.33 \mathrm{E}-03$ & $1.16 \mathrm{E}-03$ & $9.97 \mathrm{E}-04$ & $8.31 \mathrm{E}-04$ & $6.66 \mathrm{E}-04$ & $5.00 \mathrm{E}-04$ & $3.34 \mathrm{E}-04$ & $1.68 \mathrm{E}-04$ & $2.21 \mathrm{E}-06$ \\
\hline Hexachlorobutadiene & $7.00 \mathrm{E}-07$ & $1.40 \mathrm{E}-06$ & $2.10 \mathrm{E}-06$ & $2.80 \mathrm{E}-06$ & $3.50 \mathrm{E}-06$ & $4.20 \mathrm{E}-06$ & $4.90 \mathrm{E}-06$ & $5.60 \mathrm{E}-06$ & $6.30 \mathrm{E}-06$ & $7.00 \mathrm{E}-06$ & $7.70 \mathrm{E}-06$ & $8.40 \mathrm{E}-06$ \\
\hline Hexachlorocyclopentadiene & NA & NA & NA & NA & NA & NA & NA & NA & NA & NA & NA & NA \\
\hline Hexachloroethane & $1.61 \mathrm{E}-02$ & $1.53 \mathrm{E}-02$ & $1.46 \mathrm{E}-02$ & $1.30 \mathrm{E}-02$ & $1.14 \mathrm{E}-02$ & $9.82 \mathrm{E}-03$ & $8.24 \mathrm{E}-03$ & $6.66 \mathrm{E}-03$ & $5.08 \mathrm{E}-03$ & $3.50 \mathrm{E}-03$ & $1.92 \mathrm{E}-03$ & $3.35 \mathrm{E}-04$ \\
\hline Hexane & NA & NA & NA & NA & NA & NA & NA & NA & NA & NA & NA & NA \\
\hline Hydrochloric Acid & $8.23 \mathrm{E}-01$ & $9.24 \mathrm{E}-01$ & $9.76 \mathrm{E}-01$ & $1.06 \mathrm{E}+00$ & $1.12 \mathrm{E}+00$ & $1.21 \mathrm{E}+00$ & $1.31 \mathrm{E}+00$ & $1.32 \mathrm{E}+00$ & $1.29 \mathrm{E}+00$ & $1.27 \mathrm{E}+00$ & $1.26 \mathrm{E}+00$ & $1.26 \mathrm{E}+00$ \\
\hline Isophorone & NA & NA & NA & NA & NA & NA & NA & NA & NA & NA & NA & NA \\
\hline Lead & $2.66 \mathrm{E}-03$ & $4.38 \mathrm{E}-03$ & $6.08 \mathrm{E}-03$ & $7.76 \mathrm{E}-03$ & $9.43 \mathrm{E}-03$ & $1.11 \mathrm{E}-02$ & $1.28 \mathrm{E}-02$ & $1.45 \mathrm{E}-02$ & $1.61 \mathrm{E}-02$ & $1.78 \mathrm{E}-02$ & $1.95 \mathrm{E}-02$ & $2.12 \mathrm{E}-02$ \\
\hline Manganese & $4.00 \mathrm{E}-04$ & $4.28 \mathrm{E}-04$ & $4.50 \mathrm{E}-04$ & $4.55 \mathrm{E}-04$ & $4.53 \mathrm{E}-04$ & $4.58 \mathrm{E}-04$ & $4.61 \mathrm{E}-04$ & $4.63 \mathrm{E}-04$ & 4.63E-04 & $4.73 \mathrm{E}-04$ & 4.77E-04 & $4.84 \mathrm{E}-04$ \\
\hline Mercury & 7.04E-04 & $8.47 \mathrm{E}-04$ & $8.29 \mathrm{E}-04$ & $8.32 \mathrm{E}-04$ & $8.31 \mathrm{E}-04$ & $8.34 \mathrm{E}-04$ & $8.35 \mathrm{E}-04$ & $8.37 \mathrm{E}-04$ & $8.37 \mathrm{E}-04$ & $8.42 \mathrm{E}-04$ & $8.45 \mathrm{E}-04$ & $8.48 \mathrm{E}-04$ \\
\hline Methanol & $3.14 \mathrm{E}-02$ & $3.02 \mathrm{E}-02$ & $2.89 \mathrm{E}-02$ & $2.59 \mathrm{E}-02$ & $2.30 \mathrm{E}-02$ & $2.00 \mathrm{E}-02$ & $1.70 \mathrm{E}-02$ & $1.41 \mathrm{E}-02$ & $1.11 \mathrm{E}-02$ & 8.09E-03 & $5.11 \mathrm{E}-03$ & $2.14 \mathrm{E}-03$ \\
\hline Methyl isobutyl ketone & $3.09 \mathrm{E}-02$ & $2.95 \mathrm{E}-02$ & $2.81 \mathrm{E}-02$ & $2.50 \mathrm{E}-02$ & $2.19 \mathrm{E}-02$ & $1.87 \mathrm{E}-02$ & $1.56 \mathrm{E}-02$ & $1.25 \mathrm{E}-02$ & $9.37 \mathrm{E}-03$ & $6.27 \mathrm{E}-03$ & $3.18 \mathrm{E}-03$ & $9.04 \mathrm{E}-05$ \\
\hline Methylene chloride & $8.16 \mathrm{E}-02$ & $7.47 \mathrm{E}-02$ & $6.74 \mathrm{E}-02$ & $6.02 \mathrm{E}-02$ & $5.30 \mathrm{E}-02$ & $4.58 \mathrm{E}-02$ & $3.86 \mathrm{E}-02$ & $3.14 \mathrm{E}-02$ & $2.43 \mathrm{E}-02$ & $1.71 \mathrm{E}-02$ & $9.91 \mathrm{E}-03$ & $2.72 \mathrm{E}-03$ \\
\hline Naphthalene & $1.32 \mathrm{E}-03$ & $1.37 \mathrm{E}-03$ & $1.41 \mathrm{E}-03$ & $1.42 \mathrm{E}-03$ & $1.45 \mathrm{E}-03$ & $1.47 \mathrm{E}-03$ & $1.46 \mathrm{E}-03$ & $1.42 \mathrm{E}-03$ & $1.38 \mathrm{E}-03$ & $1.39 \mathrm{E}-03$ & $1.40 \mathrm{E}-03$ & $1.51 \mathrm{E}-03$ \\
\hline Nickel & $4.18 \mathrm{E}-03$ & $4.20 \mathrm{E}-03$ & $4.21 \mathrm{E}-03$ & $4.21 \mathrm{E}-03$ & $4.21 \mathrm{E}-03$ & $4.21 \mathrm{E}-03$ & $4.21 \mathrm{E}-03$ & $4.22 \mathrm{E}-03$ & $4.22 \mathrm{E}-03$ & $4.22 \mathrm{E}-03$ & $4.22 \mathrm{E}-03$ & $4.23 \mathrm{E}-03$ \\
\hline Nitrobenzene & $3.12 \mathrm{E}-02$ & $2.98 \mathrm{E}-02$ & $2.85 \mathrm{E}-02$ & $2.54 \mathrm{E}-02$ & $2.24 \mathrm{E}-02$ & $1.93 \mathrm{E}-02$ & $1.62 \mathrm{E}-02$ & $1.32 \mathrm{E}-02$ & $1.01 \mathrm{E}-02$ & $7.04 \mathrm{E}-03$ & $3.98 \mathrm{E}-03$ & $9.21 \mathrm{E}-04$ \\
\hline N-Nitrosodimethylamine & NA & NA & NA & NA & NA & NA & NA & NA & NA & NA & NA & NA \\
\hline Polychlorinated biphenyls & $1.38 \mathrm{E}-02$ & $1.62 \mathrm{E}-02$ & $1.87 \mathrm{E}-02$ & $2.08 \mathrm{E}-02$ & $2.29 \mathrm{E}-02$ & $2.50 \mathrm{E}-02$ & $2.71 \mathrm{E}-02$ & $2.92 \mathrm{E}-02$ & $3.13 \mathrm{E}-02$ & $3.34 \mathrm{E}-02$ & $3.55 \mathrm{E}-02$ & $3.76 \mathrm{E}-02$ \\
\hline Pentachloronitrobenzene & NA & NA & NA & NA & NA & NA & NA & NA & NA & NA & NA & NA \\
\hline Pentachlorophenol & $3.80 \mathrm{E}-04$ & $7.59 \mathrm{E}-04$ & $1.14 \mathrm{E}-03$ & $1.52 \mathrm{E}-03$ & $1.90 \mathrm{E}-03$ & $2.28 \mathrm{E}-03$ & $2.66 \mathrm{E}-03$ & $3.04 \mathrm{E}-03$ & $3.42 \mathrm{E}-03$ & $3.80 \mathrm{E}-03$ & 4.17E-03 & $4.55 \mathrm{E}-03$ \\
\hline Phenol & NA & NA & NA & NA & NA & NA & NA & NA & NA & NA & NA & NA \\
\hline Phosphorus & NA & NA & NA & NA & NA & NA & NA & NA & NA & NA & NA & NA \\
\hline
\end{tabular}


Table A-2 (cont.)

\begin{tabular}{|c|c|c|c|c|c|c|c|c|c|c|c|c|}
\hline $\begin{array}{l}\text { Hazardous Air Pollutant } \\
\text { (Tons) }\end{array}$ & Jan-18 & Feb-18 & Mar-18 & Apr-18 & May-18 & Jun-18 & Jul-18 & Aug-18 & Sep-18 & Oct-18 & Nov-18 & Dec-18 \\
\hline POM/PAH & $2.88 \mathrm{E}-03$ & $3.10 \mathrm{E}-03$ & $3.27 \mathrm{E}-03$ & $3.35 \mathrm{E}-03$ & $3.48 \mathrm{E}-03$ & $3.56 \mathrm{E}-03$ & $3.62 \mathrm{E}-03$ & $3.61 \mathrm{E}-03$ & $3.61 \mathrm{E}-03$ & $3.69 \mathrm{E}-03$ & $3.78 \mathrm{E}-03$ & $3.97 \mathrm{E}-03$ \\
\hline Selenium & $1.07 \mathrm{E}-03$ & $1.21 \mathrm{E}-03$ & $1.33 \mathrm{E}-03$ & $1.41 \mathrm{E}-03$ & $1.48 \mathrm{E}-03$ & $1.56 \mathrm{E}-03$ & $1.63 \mathrm{E}-03$ & $1.71 \mathrm{E}-03$ & $1.77 \mathrm{E}-03$ & $1.87 \mathrm{E}-03$ & $1.95 \mathrm{E}-03$ & $2.03 E-03$ \\
\hline Styrene & $6.11 \mathrm{E}-07$ & $6.27 \mathrm{E}-07$ & $6.30 \mathrm{E}-07$ & $6.65 \mathrm{E}-07$ & $6.74 \mathrm{E}-07$ & $6.76 \mathrm{E}-07$ & $6.63 \mathrm{E}-07$ & $6.51 \mathrm{E}-07$ & $6.07 \mathrm{E}-07$ & $5.35 \mathrm{E}-07$ & $4.94 \mathrm{E}-07$ & $3.87 \mathrm{E}-07$ \\
\hline Tetrachloroethylene & $4.12 \mathrm{E}-02$ & $3.92 \mathrm{E}-02$ & $3.72 \mathrm{E}-02$ & $3.31 \mathrm{E}-02$ & $2.91 \mathrm{E}-02$ & $2.50 \mathrm{E}-02$ & $2.10 \mathrm{E}-02$ & $1.69 \mathrm{E}-02$ & $1.29 \mathrm{E}-02$ & $8.81 \mathrm{E}-03$ & $4.76 \mathrm{E}-03$ & $7.06 \mathrm{E}-04$ \\
\hline Toluene & $3.83 \mathrm{E}-01$ & $3.68 \mathrm{E}-01$ & $3.52 \mathrm{E}-01$ & $3.19 \mathrm{E}-01$ & $2.86 \mathrm{E}-01$ & $2.53 \mathrm{E}-01$ & $2.19 \mathrm{E}-01$ & $1.86 \mathrm{E}-01$ & $1.53 \mathrm{E}-01$ & $1.20 \mathrm{E}-01$ & $8.62 \mathrm{E}-02$ & $5.32 \mathrm{E}-02$ \\
\hline Trichloroethylene & $3.10 \mathrm{E}-01$ & $2.96 \mathrm{E}-01$ & $2.82 \mathrm{E}-01$ & $2.51 \mathrm{E}-01$ & $2.20 \mathrm{E}-01$ & $1.88 \mathrm{E}-01$ & $1.57 \mathrm{E}-01$ & $1.26 \mathrm{E}-01$ & $9.48 \mathrm{E}-02$ & $6.36 \mathrm{E}-02$ & $3.25 \mathrm{E}-02$ & $1.29 \mathrm{E}-03$ \\
\hline Vinyl Chloride & $4.86 \mathrm{E}-04$ & 4.64E-04 & $4.41 \mathrm{E}-04$ & $3.93 \mathrm{E}-04$ & $3.44 \mathrm{E}-04$ & $2.95 \mathrm{E}-04$ & $2.47 \mathrm{E}-04$ & $1.98 \mathrm{E}-04$ & $1.50 \mathrm{E}-04$ & 1.01E-04 & $5.23 \mathrm{E}-05$ & $3.61 \mathrm{E}-06$ \\
\hline Xylene & $4.76 \mathrm{E}-02$ & $4.61 \mathrm{E}-02$ & $4.43 \mathrm{E}-02$ & $4.04 \mathrm{E}-02$ & $3.68 \mathrm{E}-02$ & $3.26 \mathrm{E}-02$ & $2.87 \mathrm{E}-02$ & $2.42 \mathrm{E}-02$ & $2.03 \mathrm{E}-02$ & 1.67E-02 & $1.30 \mathrm{E}-02$ & $1.09 \mathrm{E}-02$ \\
\hline Total & $5.06 \mathrm{E}+00$ & $4.88 \mathrm{E}+00$ & $4.65 \mathrm{E}+00$ & $4.35 \mathrm{E}+00$ & $4.03 E+00$ & $3.74 \mathrm{E}+00$ & $3.46 \mathrm{E}+00$ & $3.08 \mathrm{E}+00$ & $2.67 \mathrm{E}+00$ & $2.28 \mathrm{E}+00$ & $1.88 \mathrm{E}+00$ & $1.49 \mathrm{E}+00$ \\
\hline Maximum HAP Emission & $1.90 \mathrm{E}+00$ & $1.73 \mathrm{E}+00$ & $1.56 \mathrm{E}+00$ & $1.39 \mathrm{E}+00$ & $1.21 \mathrm{E}+00$ & $1.21 \mathrm{E}+00$ & $1.31 \mathrm{E}+00$ & $1.32 \mathrm{E}+00$ & $1.29 \mathrm{E}+00$ & $1.27 \mathrm{E}+00$ & $1.26 \mathrm{E}+00$ & $1.26 \mathrm{E}+00$ \\
\hline Maximum HAP Emitted & Chloroform & Chloroform & Chloroform & Chloroform & Chloroform & $\begin{array}{l}\text { Hydrochloric } \\
\text { Acid }\end{array}$ & $\begin{array}{l}\text { Hydrochloric } \\
\text { Acid }\end{array}$ & $\begin{array}{l}\text { Hydrochloric } \\
\text { Acid }\end{array}$ & $\begin{array}{l}\text { Hydrochloric } \\
\text { Acid }\end{array}$ & $\begin{array}{l}\text { Hydrochloric } \\
\text { Acid }\end{array}$ & $\begin{array}{l}\text { Hydrochloric } \\
\text { Acid }\end{array}$ & $\begin{array}{l}\text { Hydrochloric } \\
\text { Acid }\end{array}$ \\
\hline
\end{tabular}

\begin{tabular}{|c|c|c|c|c|c|c|c|c|c|c|c|c|}
\hline Criteria Air Pollutant & Jan-18 & Feb-18 & Mar-18 & Apr-18 & May-18 & Jun-18 & Jul-18 & Aug-18 & Sep-18 & Oct-18 & Nov-18 & Dec-18 \\
\hline $\mathrm{SO} 2$ & $5.70 \mathrm{E}-02$ & $2.70 \mathrm{E}-01$ & 4.69E-01 & $6.66 \mathrm{E}-01$ & $2.74 \mathrm{E}-01$ & $2.01 \mathrm{E}-01$ & $1.76 \mathrm{E}-01$ & $2.15 \mathrm{E}-01$ & $1.76 \mathrm{E}-01$ & $1.90 \mathrm{E}-01$ & $1.95 \mathrm{E}+00$ & $2.16 \mathrm{E}+00$ \\
\hline NOx & $2.62 \mathrm{E}+00$ & $6.05 E+00$ & $9.12 \mathrm{E}+00$ & $1.19 \mathrm{E}+01$ & $3.84 \mathrm{E}+00$ & $2.07 \mathrm{E}+00$ & $2.06 \mathrm{E}+00$ & $2.91 \mathrm{E}+00$ & $1.66 \mathrm{E}+00$ & $2.58 \mathrm{E}+00$ & $2.93 \mathrm{E}+01$ & $3.56 \mathrm{E}+01$ \\
\hline $\mathrm{CO}$ & 7.47E-01 & $1.90 \mathrm{E}+00$ & $2.95 \mathrm{E}+00$ & $3.94 \mathrm{E}+00$ & $1.20 \mathrm{E}+00$ & $7.68 \mathrm{E}-01$ & $6.89 \mathrm{E}-01$ & $8.65 \mathrm{E}-01$ & $6.90 \mathrm{E}-01$ & $9.22 \mathrm{E}-01$ & $9.67 \mathrm{E}+00$ & $1.16 \mathrm{E}+01$ \\
\hline PM-10/2.5 \& Condensable & $3.28 \mathrm{E}-01$ & 7.27E-01 & $1.10 \mathrm{E}+00$ & $1.39 \mathrm{E}+00$ & $3.02 \mathrm{E}-01$ & $1.88 \mathrm{E}-01$ & $1.64 \mathrm{E}-01$ & $2.03 \mathrm{E}-01$ & $1.46 \mathrm{E}-01$ & $2.91 \mathrm{E}-01$ & $2.99 \mathrm{E}+00$ & $3.51 \mathrm{E}+00$ \\
\hline VOCs & $6.65 \mathrm{E}-02$ & $3.14 \mathrm{E}-01$ & $5.43 \mathrm{E}-01$ & $7.77 \mathrm{E}-01$ & $3.39 \mathrm{E}-01$ & $2.37 \mathrm{E}-01$ & $2.08 \mathrm{E}-01$ & $2.61 \mathrm{E}-01$ & $2.09 \mathrm{E}-01$ & $2.22 \mathrm{E}-01$ & $2.31 \mathrm{E}+00$ & $2.66 \mathrm{E}+00$ \\
\hline
\end{tabular}

Note 1: $\quad$ NA indicates no emissions for Calendar Year 2018. 
Appendix B

\section{Data Records}


INTENTIONALLY BLANK 
Table B-1; 2018 ICE Operations Summary

\begin{tabular}{|c|c|c|c|c|c|}
\hline Facility & Engine ID & Fuel Rate & $\begin{array}{l}\text { Rate } \\
\text { Units }\end{array}$ & $\begin{array}{c}\text { Annual } \\
\text { Duration } \\
\text { (hr) }\end{array}$ & $\begin{array}{c}\text { Annual Fuel } \\
\text { Consumption } \\
\left.\text { (gal or } \mathrm{ft}^{3}\right)\end{array}$ \\
\hline \multicolumn{6}{|l|}{ Engines $\leq 600 \mathrm{hp}$} \\
\hline AMWTP & BGEN-232-001 & 11.00 & $\mathrm{gal} / \mathrm{hr}$ & 3.2 & 35.2 \\
\hline AMWTP & BGEN-RCE-001 & 4.90 & $\mathrm{gal} / \mathrm{hr}$ & 127.7 & 625.7 \\
\hline ATR Complex & 609-M-87 & 13.10 & $\mathrm{gal} / \mathrm{hr}$ & 41.9 & 548.9 \\
\hline ATR Complex & $619-10$ & 26.00 & $\mathrm{gal} / \mathrm{hr}$ & 14.0 & 362.7 \\
\hline ATR Complex & 633-M-1 & 16.10 & $\mathrm{gal} / \mathrm{hr}$ & 12.9 & 207.7 \\
\hline ATR Complex & 680-M-1 & 11.10 & $\mathrm{gal} / \mathrm{hr}$ & 2.5 & 27.8 \\
\hline ATR Complex & 688-M-1 & 20.00 & $\mathrm{gal} / \mathrm{hr}$ & 21.6 & 432.0 \\
\hline ATR Complex & $688-M-2$ & 20.00 & $\mathrm{gal} / \mathrm{hr}$ & 10.9 & 218.0 \\
\hline CFA & TAN-687 & 3.26 & $\mathrm{gal} / \mathrm{hr}$ & 10.9 & 35.5 \\
\hline CFA & CFA-609-001 & 8.33 & $\mathrm{gal} / \mathrm{hr}$ & 41.9 & 349.0 \\
\hline CFA & CFA-1603-001 & 10.00 & $\mathrm{gal} / \mathrm{hr}$ & 96.8 & 968.0 \\
\hline CFA & CFA-1603-002 & 10.00 & $\mathrm{gal} / \mathrm{hr}$ & 70.3 & 703.0 \\
\hline CFA & PER-638-004 & 10.00 & $\mathrm{gal} / \mathrm{hr}$ & 48.5 & 485.0 \\
\hline CFA & TAN-665-002 & 14.00 & $\mathrm{gal} / \mathrm{hr}$ & 60.2 & 842.8 \\
\hline CFA & TAN-610-002 & 14.00 & $\mathrm{gal} / \mathrm{hr}$ & 31.3 & 437.8 \\
\hline CFA & B8-601 & 2.88 & $\mathrm{gal} / \mathrm{hr}$ & 3.7 & 10.7 \\
\hline CFA & B27-601 & 2.88 & $\mathrm{gal} / \mathrm{hr}$ & 12.0 & 34.6 \\
\hline CFA & CFA-668-001 & 16.40 & $\mathrm{gal} / \mathrm{hr}$ & 73.4 & 1203.8 \\
\hline CFA & ARA-632 & 7.40 & $\mathrm{gal} / \mathrm{hr}$ & 10.2 & 75.5 \\
\hline CFA & TAN-601 & 3.44 & $\mathrm{gal} / \mathrm{hr}$ & 25.7 & 88.4 \\
\hline CFA & CFA-609-002 & 4.3 & $\mathrm{gal} / \mathrm{hr}$ & 66.7 & 286.8 \\
\hline CFA & B27-607/609 & 14.9 & $\mathrm{gal} / \mathrm{hr}$ & 9.4 & 139.8 \\
\hline INTEC & MOT-YDA-202 & 4.50 & $\mathrm{gal} / \mathrm{hr}$ & 2.8 & 12.7 \\
\hline INTEC & P-UTI-608 & 17.59 & $\mathrm{gal} / \mathrm{hr}$ & 10.6 & 186.5 \\
\hline INTEC & P-UTI-673 & 19.15 & $\mathrm{gal} / \mathrm{hr}$ & 79.3 & 1518.6 \\
\hline INTEC & P-UTI-672 & 19.15 & $\mathrm{gal} / \mathrm{hr}$ & 50.0 & 957.5 \\
\hline INTEC & COM-UTI-616 & 23.80 & $\mathrm{gal} / \mathrm{hr}$ & 24.0 & 571.2 \\
\hline RWMC & EFW-ENG-4301 & 7.24 & $\mathrm{gal} / \mathrm{hr}$ & 26.0 & 188.2 \\
\hline RWMC & S GEN-RE501 & 8.12 & $\mathrm{gal} / \mathrm{hr}$ & 8.7 & 70.6 \\
\hline RWMC & EFW-ENG-3901 & 13.20 & $\mathrm{gal} / \mathrm{hr}$ & 28.3 & 373.6 \\
\hline RWMC & S-GEN-301 & 20.61 & $\mathrm{gal} / \mathrm{hr}$ & 33.4 & 688.4 \\
\hline RWMC & BA-CMP-T1101 & 1.45 & $\mathrm{gal} / \mathrm{hr}$ & 39.3 & 57.0 \\
\hline RWMC & S-GEN-T1401 & 1.93 & $\mathrm{gal} / \mathrm{hr}$ & 0.0 & 0.0 \\
\hline RWMC & S-GEN-1 & 19.00 & $\mathrm{gal} / \mathrm{hr}$ & 5.2 & 99.6 \\
\hline RWMC & HV-GEN-RE301 & 8.20 & $\mathrm{gal} / \mathrm{hr}$ & 9.0 & 73.8 \\
\hline RWMC & S-GEN-RE401 & 8.20 & $\mathrm{gal} / \mathrm{hr}$ & 19.8 & 162.4 \\
\hline RWMC & S-GEN-RE701 & 8.20 & $\mathrm{gal} / \mathrm{hr}$ & 10.5 & 86.1 \\
\hline RWMC & S-GEN-RE801 & 8.20 & $\mathrm{gal} / \mathrm{hr}$ & 486.5 & 3989.3 \\
\hline RWMC & S-GEN-RE901 & 15.50 & $\mathrm{gal} / \mathrm{hr}$ & 9.2 & 142.6 \\
\hline MFC & ANL- 798--008 & 1.70 & $\mathrm{gal} / \mathrm{hr}$ & 7.4 & 12.6 \\
\hline MFC & ANL-720-018 & 2.80 & $\mathrm{gal} / \mathrm{hr}$ & 81.8 & 229.0 \\
\hline MFC & ANL-725 & 2.80 & $\mathrm{gal} / \mathrm{hr}$ & 8.9 & 24.9 \\
\hline MFC & ANL-754-003 & 4.00 & $\mathrm{gal} / \mathrm{hr}$ & 0.0 & 0.0 \\
\hline MFC & ANL-704-015 & 4.00 & $\mathrm{gal} / \mathrm{hr}$ & 8.2 & 32.8 \\
\hline MFC & ANL-785-016 & 5.00 & $\mathrm{gal} / \mathrm{hr}$ & 3.0 & 15.0 \\
\hline MFC & ANL-701-009 & 9.40 & $\mathrm{gal} / \mathrm{hr}$ & 17.0 & 159.8 \\
\hline MFC & ANL- 774-001 & 9.00 & $\mathrm{gal} / \mathrm{hr}$ & 10.9 & 98.1 \\
\hline
\end{tabular}


Table B-1 (cont.)

\begin{tabular}{|c|c|c|c|c|c|}
\hline Facility & Engine ID & Fuel Rate & $\begin{array}{l}\text { Rate } \\
\text { Units }\end{array}$ & $\begin{array}{c}\text { Annual } \\
\text { Duration } \\
\text { (hr) }\end{array}$ & $\begin{array}{c}\text { Annual Fuel } \\
\text { Consumption } \\
\left.\text { (gal or } \mathrm{ft}^{3}\right)\end{array}$ \\
\hline MFC & ANL-720-017 & 9.00 & gal/hr & 2.3 & 20.7 \\
\hline MFC & ANL-768-028 & 9.00 & $\mathrm{gal} / \mathrm{hr}$ & 0.0 & 0.0 \\
\hline MFC & ANL-1728 & 10.40 & $\mathrm{gal} / \mathrm{hr}$ & 105.1 & 1093.0 \\
\hline MFC & ANL-752A-001 & 20.00 & $\mathrm{gal} / \mathrm{hr}$ & 21.8 & 436.0 \\
\hline MFC & ANL-756 & 22.70 & $\mathrm{gal} / \mathrm{hr}$ & 6.0 & 136.2 \\
\hline MFC & ANL-792A-002 & 21.00 & $\mathrm{gal} / \mathrm{hr}$ & 29.0 & 609.0 \\
\hline MFC & ANL-707-002 & 21.00 & $\mathrm{gal} / \mathrm{hr}$ & 27.2 & 571.2 \\
\hline MFC & ANL-709-008 & 24.00 & $\mathrm{gal} / \mathrm{hr}$ & 14.0 & 336.0 \\
\hline MFC & ANL-709-016 & 24.00 & $\mathrm{gal} / \mathrm{hr}$ & 15.0 & 360.0 \\
\hline MFC & ANL-785-017 & 28.00 & gal/hr & 8.6 & 240.8 \\
\hline MFC & ANL-787 & 11.40 & $\mathrm{gal} / \mathrm{hr}$ & 9.3 & 106.0 \\
\hline MFC & ANL-1740 & 12.00 & $\mathrm{gal} / \mathrm{hr}$ & 44.1 & 529.2 \\
\hline MFC & ANL-1729 & 13.60 & $\mathrm{gal} / \mathrm{hr}$ & 8.8 & 119.7 \\
\hline \multirow[t]{2}{*}{ SMC } & TAN 675-010 & 15.60 & $\mathrm{gal} / \mathrm{hr}$ & 52.1 & 812.8 \\
\hline & & & & Total & 23239.3 \\
\hline \multicolumn{6}{|c|}{ Liquified Natural Gas } \\
\hline \multirow[t]{2}{*}{ CFA } & CFA-1611 & 1810.00 & $\mathrm{ft} 3 / \mathrm{hr}$ & 10.7 & 19367.0 \\
\hline & & & & Total & 19367.0 \\
\hline \multicolumn{6}{|l|}{ Propane } \\
\hline AMWTP & S1-GEN-1001 & 16.80 & $\mathrm{gal} / \mathrm{hr}$ & 5.4 & 90.7 \\
\hline \multirow[t]{2}{*}{ CFA } & GE-B28601 & 8.40 & $\mathrm{gal} / \mathrm{hr}$ & 27.8 & 233.5 \\
\hline & & & & Total & 324.2 \\
\hline \multicolumn{6}{|l|}{ Engines > $600 \mathrm{hp}$} \\
\hline AMWTP & WMF-734 & 39.40 & $\mathrm{gal} / \mathrm{hr}$ & 7.0 & 275.8 \\
\hline AMWTP & BGEN-812-001 & 27.10 & $\mathrm{gal} / \mathrm{hr}$ & 5.0 & 134.1 \\
\hline AMWTP & BGEN-812-002 & 44.20 & $\mathrm{gal} / \mathrm{hr}$ & 3.8 & 168.0 \\
\hline ATR Complex & 670-M-42 & \multirow{3}{*}{ Note 1} & $\mathrm{gal} / \mathrm{hr}$ & & 0.0 \\
\hline ATR Complex & $670-M-43$ & & $\mathrm{gal} / \mathrm{hr}$ & & 13403.0 \\
\hline ATR Complex & 674-M-6 & & $\mathrm{gal} / \mathrm{hr}$ & & 1679.0 \\
\hline ATR Complex & 786-M-1 & 123.10 & $\mathrm{gal} / \mathrm{hr}$ & 29.0 & 3569.9 \\
\hline INTEC & GEN-WCS-002 & 119.23 & $\mathrm{gal} / \mathrm{hr}$ & 16.0 & 1907.7 \\
\hline INTEC & GEN-WCS-004 & 119.23 & $\mathrm{gal} / \mathrm{hr}$ & 15.0 & 1788.5 \\
\hline INTEC & GEN-WCS-006 & 119.23 & $\mathrm{gal} / \mathrm{hr}$ & 16.0 & 1907.7 \\
\hline MFC & ANL-768-003 & 32.00 & $\mathrm{gal} / \mathrm{hr}$ & 8.8 & 281.6 \\
\hline NRF & 686-016 & 69.70 & gal/hr & 16.1 & 1122.2 \\
\hline NRF & $686-017$ & 69.70 & $\mathrm{gal} / \mathrm{hr}$ & 16.9 & 1177.9 \\
\hline NRF & $686-018$ & 69.70 & $\mathrm{gal} / \mathrm{hr}$ & 12.5 & 871.3 \\
\hline NRF & $686-019$ & 69.70 & $\mathrm{gal} / \mathrm{hr}$ & 12.0 & 836.4 \\
\hline SMC & TAN 679-012 & 20.40 & $\mathrm{gal} / \mathrm{hr}$ & 3.5 & 71.4 \\
\hline & & & & Total & 29194.4 \\
\hline
\end{tabular}

Note 1: Fuel is calculated from fuel storage tank measurements. 
Table B-2; 2018 INL Boiler Summary

\begin{tabular}{|c|c|c|c|c|c|c|}
\hline & \multirow[b]{3}{*}{ Fuel Type } & \multicolumn{5}{|c|}{ Facility } \\
\hline & & CFA & \multicolumn{2}{|c|}{$\mathrm{FI}$} & \multirow{2}{*}{$\begin{array}{c}\text { NRF } \\
\text { Distillate } \\
\text { (gallons) }\end{array}$} & \multirow{2}{*}{$\begin{array}{c}\text { SMC } \\
\text { Distillate } \\
\text { (gallons) }\end{array}$} \\
\hline & & $\begin{array}{l}\text { Distillate } \\
\text { (gallons) }\end{array}$ & $\begin{array}{l}\text { Distillate } \\
\text { (gallons) }\end{array}$ & $\begin{array}{l}\text { Propane } \\
\text { (gallons) }\end{array}$ & & \\
\hline \multirow{12}{*}{$\begin{array}{l}\frac{c}{+} \\
\stackrel{\underline{\underline{C}}}{0} \\
\sum\end{array}$} & January & 3374.0 & 75850.0 & 17619.1 & 71245.0 & 23366.0 \\
\hline & February & 3317.0 & 68687.0 & 13920.0 & 64987.0 & 20937.0 \\
\hline & March & 3398.0 & 65648.0 & 9321.2 & 63615.0 & 20512.0 \\
\hline & April & 2291.0 & 48472.0 & 6507.5 & 29708.0 & 14177.0 \\
\hline & May & 66.0 & 35993.0 & 3812.8 & 1631.0 & 6328.0 \\
\hline & June & 0.0 & 27282.0 & 1750.2 & 0.0 & 0.0 \\
\hline & July & 7.0 & 28267.0 & 580.5 & 0.0 & 0.0 \\
\hline & August & 0.0 & 23919.0 & 768.6 & 0.0 & 0.0 \\
\hline & September & 0.0 & 15391.0 & 1942.1 & 1177.0 & 0.0 \\
\hline & October & 2490.0 & 44699.0 & 6253.6 & 45110.0 & 10080.0 \\
\hline & November & 3439.0 & 71053.0 & 9764.2 & 64759.0 & 21361.0 \\
\hline & December & 4056.0 & 87694.0 & 15285.9 & 80473.0 & 28015.0 \\
\hline & Total & 22438.0 & 592955.0 & 87525.8 & 422705.0 & 144776.0 \\
\hline
\end{tabular}


Table B-3; Mobile Equipment Operation Hours

TSA-RE Monthly Mobile Equipment Hours of Operation

\begin{tabular}{|c|c|c|c|c|c|c|c|c|c|c|c|c|c|c|}
\hline Equip \# & Description & Fuel & Jan & Feb & Mar & Apr & May & Jun & Jul & Aug & Sep & Oct & Nov & Dec \\
\hline Q-020-008 & Genie Manlift (Model Z45/25 DC) & Diesel & 0 & 1 & 0 & 2 & 1 & 1 & 4 & 2 & 3 & 2 & 0 & 3 \\
\hline Q-120-002-A & Gehl Dynalift & Diesel & 13 & 0 & 0 & 13 & 3 & 2 & 4 & 1 & 3 & 2 & 2 & 3 \\
\hline Q-120-002-B & Gehl Dynalift & Diesel & 0 & 0 & 0 & 0 & 2 & 1 & 0 & 0 & 1 & 1 & 1 & 0 \\
\hline Q-042-002-B & Hyster Forklift (Model H50XM) & Propane & 4 & 0 & 0 & 0 & 0 & 0 & 0 & 0 & 0 & 0 & 0 & 0 \\
\hline Q-042-002-C & Hyster Forklift (Model H50XM) & Propane & 1 & 8 & 20 & 20 & 5 & 3 & 0 & 7 & 4 & 9 & 11 & 3 \\
\hline Q-042-002-D & Hyster Forklift (Model H50XM) & Propane & 4 & 2 & 0 & 28 & 5 & 1 & 1 & 2 & 4 & 4 & 6 & 1 \\
\hline Q-042-003-B & Hyster Forklift (Model H80XM) & Propane & 13 & 4 & 6 & 15 & 15 & 12 & 14 & 3 & 8 & 5 & 2 & 6 \\
\hline Q-180-102 & Caterpillar GP-45K Forklift & Propane & 2 & 1 & 0 & 0 & 0 & 1 & 4 & 0 & 0 & 1 & 0 & 0 \\
\hline
\end{tabular}




\section{Appendix C}

\section{Updated Equipment List}


INTENTIONALLY BLANK 
Table C-1; Idaho National Laboratory Engine Information

\begin{tabular}{|c|c|c|c|c|c|c|c|c|c|c|c|c|c|c|}
\hline Facility & Engine ID & Type $^{a}$ & $\begin{array}{c}\text { New/ } \\
\text { Existing }\end{array}$ & NSPS & $\begin{array}{l}\text { Model } \\
\text { Year }\end{array}$ & $\begin{array}{l}\text { Installation } \\
\text { Date }\end{array}$ & $\begin{array}{c}\operatorname{Max} \\
\mathrm{HP}\end{array}$ & $\begin{array}{l}\text { Tier } \\
\text { Cert }\end{array}$ & $\begin{array}{l}\text { Ignition } \\
\text { Type }\end{array}$ & $\begin{array}{l}\text { Fuel } \\
\text { Type }^{b}\end{array}$ & $\begin{array}{c}\text { Fuel } \\
\text { Rate } \\
(\text { gal } / \mathrm{hr})^{\mathrm{c}}\end{array}$ & $\begin{array}{l}\text { Removed } \\
\text { from } \\
\text { Service }\end{array}$ & $\begin{array}{l}\text { Included in } \\
\text { Application }\end{array}$ & $\begin{array}{l}\text { In Service } \\
\text { at Issuance }\end{array}$ \\
\hline AMWTP & S1-GEN 1001 & ESG & $\mathrm{E}$ & NA & 2001 & 2002 & 225 & NA & $\mathrm{SI}$ & Propane & 16.8 & NA & Yes & Yes \\
\hline AMWTP & BGEN-232-001 & ESG & $E$ & NA & 2001 & 2002 & 380 & NA & $\mathrm{Cl}$ & Distillate & 11 & NA & Yes & Yes \\
\hline AMWTPf & $\begin{array}{c}\text { BGEN-RCE- } \\
001\end{array}$ & $A C$ & $\mathrm{~N}$ & Subpart IIII & 2008 & 2014 & 115 & 3 & $\mathrm{Cl}$ & Distillate & 4.9 & NA & No & No \\
\hline AMWTP & BGEN-812-001 & ESG & E & NA & 2001 & 2002 & 755 & NA & $\mathrm{Cl}$ & Distillate & 27.1 & NA & Yes & Yes \\
\hline AMWTP & BGEN-812-002 & ESG & E & NA & 2002 & 2002 & 900 & NA & $\mathrm{Cl}$ & Distillate & 44.2 & NA & Yes & Yes \\
\hline AMWTP & WMF-734 & ESG & $\mathrm{E}$ & NA & 1987 & 2001 & 745 & NA & $\mathrm{Cl}$ & Distillate & 39.4 & NA & Yes & Yes \\
\hline ATR Complex & 609-M-87 & EAC & $E$ & NA & 1988 & 1998 & 250 & $N / A$ & $\mathrm{Cl}$ & Distillate & 13.1 & NA & Yes & Yes \\
\hline ATR Complex & $619-10$ & EFW & E & NA & - & 1996 & 558 & N/A & $\mathrm{Cl}$ & Distillate & 26 & NA & Yes & Yes \\
\hline ATR Complex & 633-M-1 & EFW & $\mathrm{N}$ & Subpart IIII & 2009 & 2012 & 315 & 3 & $\mathrm{Cl}$ & Distillate & $\sim 16.1^{\mathrm{e}}$ & NA & Yes & Yes \\
\hline ATR Complex & 670-M-42 & ESG & E & NA & 1963 & 1967 & 2118 & N/A & $\mathrm{Cl}$ & Distillate & $44^{d}$ & NA & Yes & Yes \\
\hline ATR Complex & 670-M-43 & ESG & $E$ & NA & 1963 & 1967 & 2118 & $N / A$ & $\mathrm{Cl}$ & Distillate & $44^{d}$ & NA & Yes & Yes \\
\hline ATR Complex & 674-M-6 & ESG & $\mathrm{E}$ & NA & 1984 & 1985 & 2132 & N/A & $\mathrm{Cl}$ & Distillate & $44^{d}$ & NA & Yes & Yes \\
\hline ATR Complex & 680-M-1 & ESG & $\mathrm{E}$ & NA & 1991 & 1991 & 250 & $\mathrm{~N} / \mathrm{A}$ & $\mathrm{Cl}$ & Distillate & 11.1 & NA & Yes & Yes \\
\hline ATR Complex & 688-M-1 & EFW & E & NA & 1999 & 2000 & 368 & $N / A$ & $\mathrm{Cl}$ & Distillate & 20 & NA & Yes & Yes \\
\hline ATR Complex & 688-M-2 & EFW & E & NA & 1999 & 2000 & 368 & N/A & $\mathrm{Cl}$ & Distillate & 20 & NA & Yes & Yes \\
\hline ATR Complex & 786-M-1 & ESG & E & NA & 2001 & 2005 & 2593 & 1 & $\mathrm{Cl}$ & Distillate & 123.1 & NA & Yes & Yes \\
\hline CFA & CFA-609-001 & ESG & $\mathrm{E}$ & NA & 1982 & 1987 & 166 & NA & $\mathrm{Cl}$ & Distillate & $\sim 8.33$ & NA & Yes & Yes \\
\hline CFA & CFA-668-001 & ESG & $\mathrm{N}$ & Subpart IIII & 2010 & 2010 & 345 & 3 & $\mathrm{Cl}$ & Distillate & 16.4 & NA & Yes & Yes \\
\hline CFA & CFA-1603-001 & EFW & $E$ & NA & 1994 & 1994 & 196 & NA & $\mathrm{Cl}$ & Distillate & $\sim 10$ & NA & Yes & Yes \\
\hline CFA & CFA-1603-002 & EFW & E & NA & 1994 & 1994 & 196 & NA & $\mathrm{Cl}$ & Distillate & $\sim 10$ & NA & Yes & Yes \\
\hline CFA & CFA-1611 & ESG & $\mathrm{E}$ & NA & 1995 & 1996 & 220 & NA & $\mathrm{SI}$ & LNG & $\begin{array}{l}1810 \\
\mathrm{ft}^{3} / \mathrm{hr}\end{array}$ & NA & Yes & Yes \\
\hline CFA & PER-638-004 & EFW & E & NA & 1994 & 1994 & 196 & NA & $\mathrm{Cl}$ & Distillate & $\sim 10$ & NA & Yes & Yes \\
\hline CFA & GE-B28601 & ESG & E & NA & 1995 & 1996 & 61 & NA & $\mathrm{SI}$ & Propane & 8.42 & NA & Yes & Yes \\
\hline GFA ${ }^{9}$ & TAN-601 & ESG & E & NA & 2006 & 2007 & 55 & 1 & St & Propane & 6.11 & 2017 & Yes & Yes \\
\hline CFA & TAN-610-002 & EFW & $\mathrm{E}$ & NA & 1978 & 1980 & 310 & NA & $\mathrm{Cl}$ & Distillate & 14 & NA & Yes & Yes \\
\hline
\end{tabular}




\begin{tabular}{|c|c|c|c|c|c|c|c|c|c|c|c|c|c|c|}
\hline Facility & Engine ID & Type $^{a}$ & $\begin{array}{l}\text { New/ } \\
\text { Existing }\end{array}$ & NSPS & $\begin{array}{l}\text { Model } \\
\text { Year }\end{array}$ & $\begin{array}{l}\text { Installation } \\
\text { Date }\end{array}$ & $\begin{array}{c}\text { Max } \\
\text { HP }\end{array}$ & $\begin{array}{l}\text { Tier } \\
\text { Cert }\end{array}$ & $\begin{array}{l}\text { Ignition } \\
\text { Type }\end{array}$ & $\begin{array}{l}\text { Fuel } \\
\text { Type }^{\mathrm{b}}\end{array}$ & $\begin{array}{c}\text { Fuel } \\
\text { Rate } \\
\text { (gal/hr) }^{c} \\
\end{array}$ & $\begin{array}{l}\text { Removed } \\
\text { from } \\
\text { Service }\end{array}$ & $\begin{array}{l}\text { Included in } \\
\text { Application }\end{array}$ & $\begin{array}{l}\text { In Service } \\
\text { at Issuance }\end{array}$ \\
\hline CFA & TAN-665-002 & EFW & E & NA & 1979 & 1981 & 310 & NA & $\mathrm{Cl}$ & Distillate & 14 & NA & Yes & Yes \\
\hline CFA & TAN-687 & ESG & E & NA & 1988 & 1990 & 66 & NA & $\mathrm{Cl}$ & Distillate & $\sim 3.26$ & NA & Yes & Yes \\
\hline GEA & B8-601 & ESG & E & NA & 1984 & 1984 & 61 & NA & GI & Distillate & 3.12 & 2016 & Yes & Yes \\
\hline CFA & B8-601 & ESG & $\mathrm{N}$ & Subpart IIII & 2015 & 2016 & 69 & 3 & $\mathrm{Cl}$ & Distillate & 2.88 & NA & No & No \\
\hline CFA & B27-601 & ESG & $\mathrm{N}$ & Subpart IIII & 2015 & 2016 & 69 & 3 & $\mathrm{Cl}$ & Distillate & 2.88 & NA & No & No \\
\hline CFA & ARA-632 & ESG & $\mathrm{N}$ & Subpart IIII & 2015 & 2016 & 217 & 3 & $\mathrm{Cl}$ & Distillate & 7.40 & NA & No & No \\
\hline CFA & CFA-609-002 & ESG & $\mathrm{N}$ & Subpart IIII & 2012 & 2016 & 99 & 3 & $\mathrm{Cl}$ & Distillate & 4.30 & NA & No & No \\
\hline CFA & TAN-601 & ESG & $\mathrm{N}$ & Subpart IIII & 2015 & 2017 & 69 & 3 & $\mathrm{Cl}$ & Distillate & 3.44 & NA & No & No \\
\hline CFA & B27-607/609 & ESG & $\mathrm{N}$ & Subpart IIII & 2017 & 2018 & 320 & $4 \mathrm{~F}$ & $\mathrm{Cl}$ & Distillate & 14.90 & NA & No & No \\
\hline INTEC & P-UTI-673 & EFW & $E$ & NA & 1991 & 1991 & 370 & NA & $\mathrm{Cl}$ & Distillate & 19.15 & NA & Yes & Yes \\
\hline INTEC & P-UTI-608 & ESP & $E$ & NA & 1983 & 1984 & 340 & NA & $\mathrm{Cl}$ & Distillate & 17.59 & NA & Yes & Yes \\
\hline INTEC & P-UTI-672 & EFW & $E$ & NA & 1991 & 1991 & 370 & NA & $\mathrm{Cl}$ & Distillate & 19.15 & NA & Yes & Yes \\
\hline INTEC & COM-UTI-616 & EAC & E & NA & 1997 & 1997 & 460 & NA & $\mathrm{Cl}$ & Distillate & 23.8 & NA & Yes & Yes \\
\hline INTEC & GEN-WCS-002 & ESG & $\mathrm{E}$ & NA & 2000 & 2000 & 2304 & 1 & $\mathrm{Cl}$ & Distillate & 119.23 & NA & Yes & Yes \\
\hline INTEC & GEN-WCS-004 & ESG & $E$ & NA & 2000 & 2000 & 2304 & 1 & $\mathrm{Cl}$ & Distillate & 119.23 & NA & Yes & Yes \\
\hline INTEC & GEN-WCS-006 & ESG & E & NA & 2000 & 2000 & 2304 & 1 & $\mathrm{Cl}$ & Distillate & 119.23 & NA & Yes & Yes \\
\hline INTEC & MOT-YDA-202 & ESP & $E$ & NA & 1988 & 1989 & 87 & NA & $\mathrm{Cl}$ & Distillate & 4.5 & NA & Yes & Yes \\
\hline RWMC & FW-ENG-3901 & EFW & E & NA & 1980 & 1980 & 255 & NA & $\mathrm{Cl}$ & Distillate & 13.2 & NA & Yes & Yes \\
\hline RWMC & FW-ENG-4301 & EFW & $\mathrm{N}$ & Subpart IIII & 2007 & 2007 & 140 & 3 & $\mathrm{Cl}$ & Distillate & 7.24 & NA & Yes & Yes \\
\hline RWMC & S-GEN-301 & ESG & $\mathrm{N}$ & Subpart IIII & 2011 & 2011 & 398 & 3 & $\mathrm{Cl}$ & Distillate & 20.61 & NA & Yes & Yes \\
\hline RWMC & S-GEN-RE501 & ESG & $\mathrm{N}$ & Subpart IIII & 2010 & $\begin{array}{c}2010- \\
\text { CERCLA } \\
2012- \\
\text { non- } \\
\text { CERCLA } \\
\end{array}$ & 157 & 3 & $\mathrm{Cl}$ & Distillate & 8.12 & NA & Yes & Yes \\
\hline RWMC & $\begin{array}{c}\text { BA-CMP- } \\
\text { T1101 }\end{array}$ & EAC & $\mathrm{N}$ & Subpart IIII & 2007 & $\begin{array}{c}2008- \\
\text { CERCLA } \\
2012- \\
\text { non- } \\
\text { CERCLA } \\
\end{array}$ & 28 & 2 & $\mathrm{Cl}$ & Distillate & 1.45 & NA & Yes & Yes \\
\hline
\end{tabular}




\begin{tabular}{|c|c|c|c|c|c|c|c|c|c|c|c|c|c|c|}
\hline Facility & Engine ID & Type $^{a}$ & $\begin{array}{c}\text { New/ } \\
\text { Existing }\end{array}$ & NSPS & $\begin{array}{l}\text { Model } \\
\text { Year }\end{array}$ & $\begin{array}{l}\text { Installation } \\
\text { Date }\end{array}$ & $\begin{array}{c}\operatorname{Max} \\
\mathrm{HP}\end{array}$ & $\begin{array}{l}\text { Tier } \\
\text { Cert }\end{array}$ & $\begin{array}{l}\text { Ignition } \\
\text { Type }\end{array}$ & $\begin{array}{l}\text { Fuel } \\
\text { Type }^{b}\end{array}$ & $\begin{array}{c}\text { Fuel } \\
\text { Rate } \\
(\text { (gal/hr) } \\
\end{array}$ & $\begin{array}{l}\text { Removed } \\
\text { from } \\
\text { Service }\end{array}$ & $\begin{array}{l}\text { Included in } \\
\text { Application }\end{array}$ & $\begin{array}{l}\text { In Service } \\
\text { at Issuance }\end{array}$ \\
\hline RWMC & S-GEN-T1401 & ESG & $\mathrm{N}$ & $\begin{array}{c}\text { Subpart } \\
\text { JJJJ }\end{array}$ & 2010 & 2010 & 15 & 2 & $\mathrm{SI}$ & Propane & NA & NA & Yes & Yes \\
\hline RWMC & $\begin{array}{c}\text { HV-GEN- } \\
\text { RE301 }\end{array}$ & ESG & $\mathrm{N}$ & Subpart IIII & 2007 & 2007 & 364 & 3 & $\mathrm{Cl}$ & Distillate & 8.2 & NA & No & No \\
\hline RWMC & S-GEN-RE401 & ESG & $\mathrm{N}$ & Subpart IIII & 2008 & 2008 & 364 & 3 & $\mathrm{Cl}$ & Distillate & 8.2 & NA & No & No \\
\hline RWMC & S-GEN-RE701 & ESG & $\mathrm{N}$ & Subpart IIII & 2011 & 2011 & 364 & 3 & $\mathrm{Cl}$ & Distillate & 8.2 & NA & No & No \\
\hline RWMC & S-GEN-RE801 & ESG & N & Subpart IIII & 2010 & 2010 & 364 & 3 & $\mathrm{Cl}$ & Distillate & 8.2 & NA & No & No \\
\hline RWMC & S-GEN-RE901 & ESG & $\mathrm{N}$ & Subpart IIII & 2017 & 2017 & 234 & 3 & $\mathrm{Cl}$ & Distillate & 15.5 & NA & No & No \\
\hline MFC & ANL-701 & ESG & $\mathrm{E}$ & NA & 1997 & 1997 & 143 & NA & $\mathrm{Cl}$ & Distillate & 9.4 & NA & Yes & Yes \\
\hline MFC & ANL-704 & ESG & $E$ & NA & 1986 & 1986 & 86 & NA & $\mathrm{Cl}$ & Distillate & 4 & NA & Yes & Yes \\
\hline MFC & ANL-707-002 & EFW & $E$ & NA & 1990 & 1990 & 460 & NA & $\mathrm{Cl}$ & Distillate & 21 & NA & Yes & Yes \\
\hline MFC & ANL-709-008 & ESG & $E$ & NA & 1993 & 1993 & 475 & NA & $\mathrm{Cl}$ & Distillate & 24 & NA & Yes & Yes \\
\hline MFC & ANL-709-016 & ESG & $\mathrm{E}$ & NA & 1993 & 1993 & 475 & NA & $\mathrm{Cl}$ & Distillate & 24 & NA & Yes & Yes \\
\hline MFC & ANL-720-17 & ESG & $\mathrm{E}$ & NA & 1981 & 1981 & 173 & NA & $\mathrm{Cl}$ & Distillate & $\sim 9.0$ & NA & Yes & Yes \\
\hline MFC & ANL-720-18 & ESG & $\mathrm{E}$ & NA & 1980 & 1980 & 46 & NA & $\mathrm{Cl}$ & Distillate & $\sim 2.8$ & NA & Yes & Yes \\
\hline MFC & ANL-725 & ESG & $\mathrm{E}$ & NA & 1998 & 1998 & 46 & NA & $\mathrm{Cl}$ & Distillate & 2.8 & NA & Yes & Yes \\
\hline MFC & ANL-752A-001 & ESG & $\mathrm{E}$ & NA & 1989 & 1990 & 390 & NA & $\mathrm{Cl}$ & Distillate & $\sim 20$ & NA & Yes & Yes \\
\hline MFC & ANL-754 & EFW & $E$ & NA & 1960 & $\sim 1960$ & 77 & NA & $\mathrm{Cl}$ & Distillate & $\sim 4.0$ & NA & Yes & Yes \\
\hline MFC & ANL-756 & ESG & $E$ & NA & 2005 & 2006 & 450 & NA & $\mathrm{Cl}$ & Distillate & 22.7 & NA & Yes & Yes \\
\hline MFC & ANL-768-003 & ESG & $E$ & NA & 1950 & $\sim 1950$ & 741 & NA & $\mathrm{Cl}$ & Distillate & -32 & NA & Yes & Yes \\
\hline MFC & ANL-768-028 & ESG & $\mathrm{E}$ & NA & 1981 & 1981 & 173 & NA & $\mathrm{Cl}$ & Distillate & $\sim 9.0$ & NA & Yes & Yes \\
\hline MFC & ANL- 774-001 & ESG & $\mathrm{E}$ & NA & 1973 & 1973 & 166 & NA & $\mathrm{Cl}$ & Distillate & $\sim 9.0$ & NA & Yes & Yes \\
\hline MFC & ANL-785-016 & ESG & $E$ & NA & 1975 & 1975 & 110 & NA & $\mathrm{Cl}$ & Distillate & 5 & NA & Yes & Yes \\
\hline MFC & ANL-785-017 & ESG & $E$ & NA & 1950 & $\sim 1950$ & 525 & NA & $\mathrm{Cl}$ & Distillate & -28 & NA & Yes & Yes \\
\hline MFC & ANL-787 & ESG & $E$ & NA & 2008 & 2013 & 286 & NA & $\mathrm{Cl}$ & Distillate & 10.6 & NA & Yes & Yes \\
\hline MFC & ANL-792A-002 & ESG & $\mathrm{E}$ & NA & 2003 & 2004 & 450 & NA & $\mathrm{Cl}$ & Distillate & 21 & NA & Yes & Yes \\
\hline MFC & ANL- 798--008 & ESG & $\mathrm{E}$ & NA & 1981 & 1981 & 27 & NA & $\mathrm{Cl}$ & Distillate & 1.7 & NA & Yes & Yes \\
\hline MFC & ANL-1728 & ESG & $\mathrm{N}$ & Subpart IIII & 2011 & 2013 & 230 & 2 & $\mathrm{Cl}$ & Distillate & 10.4 & NA & Yes & Yes \\
\hline
\end{tabular}




\begin{tabular}{|c|c|c|c|c|c|c|c|c|c|c|c|c|c|c|}
\hline Facility & Engine ID & Type $^{a}$ & $\begin{array}{l}\text { New/ } \\
\text { Existing }\end{array}$ & NSPS & $\begin{array}{l}\text { Model } \\
\text { Year }\end{array}$ & $\begin{array}{l}\text { Installation } \\
\text { Date }\end{array}$ & $\begin{array}{l}\operatorname{Max} \\
\mathrm{HP}\end{array}$ & $\begin{array}{l}\text { Tier } \\
\text { Cert }\end{array}$ & $\begin{array}{l}\text { Ignition } \\
\text { Type }\end{array}$ & $\begin{array}{l}\text { Fuel } \\
\text { Type }^{b}\end{array}$ & $\begin{array}{c}\text { Fuel } \\
\text { Rate } \\
(\text { gal/hr) } \\
\end{array}$ & $\begin{array}{c}\text { Removed } \\
\text { from } \\
\text { Service }\end{array}$ & $\begin{array}{l}\text { Included in } \\
\text { Application }\end{array}$ & $\begin{array}{l}\text { In Service } \\
\text { at Issuance }\end{array}$ \\
\hline MFC & ANL-1740 & ESG & $\mathrm{N}$ & Subpart IIII & 2011 & 2015 & 237 & 3 & $\mathrm{Cl}$ & Distillate & 12 & NA & No & Yes \\
\hline MFC & ANL-1729 & ESG & $\mathrm{N}$ & Subpart IIII & 2016 & 2017 & 235 & 3 & $\mathrm{Cl}$ & Distillate & 13.6 & NA & No & Yes \\
\hline NRF & NRF-686-016 & ESG & $\mathrm{E}$ & NA & 1990 & 1991 & 1,443 & NA & $\mathrm{Cl}$ & Distillate & 69.7 & NA & Yes & Yes \\
\hline NRF & NRF-686-017 & ESG & E & NA & 1990 & 1991 & 1,443 & NA & $\mathrm{Cl}$ & Distillate & 69.7 & NA & Yes & Yes \\
\hline NRF & NRF-686-018 & ESG & E & NA & 1990 & 1991 & 1,443 & NA & $\mathrm{Cl}$ & Distillate & 69.7 & NA & Yes & Yes \\
\hline NRF & NRF-686-019 & ESG & $E$ & NA & 1990 & 1991 & 1,443 & NA & $\mathrm{Cl}$ & Distillate & 69.7 & NA & Yes & Yes \\
\hline SMC & TAN 675-010 & ESG & $E$ & NA & 1984 & 1984 & 598 & NA & $\mathrm{Cl}$ & Distillate & 30.9 & NA & Yes & Yes \\
\hline SMC & TAN 679-012 & ESG & $E$ & NA & 1985 & 1986 & 890 & NA & $\mathrm{Cl}$ & Distillate & 44.8 & NA & Yes & Yes \\
\hline \multirow[t]{7}{*}{ Notes: } & \multicolumn{14}{|c|}{$\begin{array}{l}\text { a. ESG = Emergency Standby Generator, EFW = Emergency Fire Water Pump, EAC = Emergency Air Compressor, ESP = Emergency Standby Pump, AC = Air } \\
\text { Compressor, G = Generator, FW = Fire Water Pump. }\end{array}$} \\
\hline & \multicolumn{14}{|c|}{ b. Distillate = \#1 or \#2 Distillate Fuel Oil with 15 ppm maximum sulfur content, LNG = Liquefied Natural Gas } \\
\hline & \multicolumn{14}{|c|}{ c. Maximum hourly fuel consumption rate } \\
\hline & \multicolumn{14}{|c|}{$\begin{array}{l}\text { d. The fuel rate listed is the actual average fuel rate for the Advanced Test Reactor Complex (ATR) ESG units 670-M-42, 670-M-43, and 674-M-6. These units do not } \\
\text { have loads connected to them that are capable of using the maximum design fuel rates (106 gph, } 106 \mathrm{gph} \text {, and } 108.1 \mathrm{gph} \text { respectively). }\end{array}$} \\
\hline & \multicolumn{14}{|c|}{ e. $\sim$ signifies that the data was estimated. } \\
\hline & \multicolumn{14}{|c|}{ f. Green highlighted entries indicate engines added after permit application submittal. } \\
\hline & \multicolumn{14}{|c|}{ g. Red highlighted entries with strike-through text entries indicate engines removed from service. } \\
\hline
\end{tabular}


Table C-2; Idaho National Laboratory Boiler Information

\begin{tabular}{|c|c|c|c|c|c|c|c|c|c|c|c|c|c|}
\hline \multirow[b]{2}{*}{ Facility } & \multicolumn{2}{|c|}{$\begin{array}{c}\begin{array}{c}\text { Use of Boiler } \\
(\%)\end{array} \\
\end{array}$} & \multirow[b]{2}{*}{ Boiler ID } & \multirow{2}{*}{$\begin{array}{c}\text { Rated } \\
\text { Capacity } \\
\text { (MMBtu/hr) }\end{array}$} & \multirow[b]{2}{*}{$\begin{array}{c}\text { Installation } \\
\text { Date }\end{array}$} & \multirow[b]{2}{*}{$\begin{array}{l}\text { Control } \\
\text { Device }\end{array}$} & \multirow[b]{2}{*}{ Fuel Type } & \multirow{2}{*}{$\begin{array}{l}\text { Full Load } \\
\text { Consumption } \\
\text { Rate } \\
\text { (gal/hr) }\end{array}$} & \multirow{2}{*}{$\begin{array}{c}\text { Actual } \\
\text { Consumption } \\
\text { Rate } \\
\text { (gal/hr) }\end{array}$} & \multirow{2}{*}{$\begin{array}{c}\text { NSPS } \\
\text { Applicability }\end{array}$} & \multirow{2}{*}{$\begin{array}{c}\text { Date } \\
\text { Removed } \\
\text { from } \\
\text { Service }\end{array}$} & \multirow[b]{2}{*}{$\begin{array}{l}\text { Included in } \\
\text { Application }\end{array}$} & \multirow[b]{2}{*}{$\begin{array}{l}\text { In Service } \\
\text { at Issuance }\end{array}$} \\
\hline & $\begin{array}{c}\text { Space } \\
\text { Heat }\end{array}$ & Process & & & & & & & & & & & \\
\hline AMWTP & 100 & 0 & WMF-676-004A & 12.55 & $6 / 7 / 2002$ & $\mathrm{O}_{2}$ Trim & Propane & 138.7 & 80.0 & Dc & NA & Yes & Yes \\
\hline AMWTP & 100 & 0 & WMF-676-005B & 12.55 & $6 / 7 / 2002$ & $\mathrm{O}_{2}$ Trim & Propane & 138.7 & 80.0 & $\mathrm{Dc}$ & NA & Yes & Yes \\
\hline AMWTP & 100 & 0 & WMF-676-006C & 12.55 & $6 / 7 / 2002$ & $\mathrm{O}_{2}$ Trim & Propane & 138.7 & 80.0 & $\mathrm{Dc}$ & NA & Yes & Yes \\
\hline AMWTP & 100 & 0 & WMF-676-007 & 2.0 & $6 / 7 / 2002$ & $\mathrm{O}_{2}$ Trim & Propane & 22.1 & 13.0 & $\mathrm{Dc}$ & NA & Yes & Yes \\
\hline CFA & 100 & 0 & CFA 608-001 & 1.5 & 1985 & O2 Trim & \#2 Diesel & 10.7 & 0.75 & NA & NA & Yes & Yes \\
\hline CFA & 100 & 0 & CFA 609-005 & 2.1 & 1987 & O2 Trim & \#2 Diesel & 15.0 & 5.8 & NA & NA & Yes & Yes \\
\hline INTEC & 48 & 52 & СРP-606-061 & 36.4 & 2000 & $\mathrm{O}_{2}$ Trim & \#2 Diesel & 216 & 32.3 & $\mathrm{Dc}$ & NA & Yes & Yes \\
\hline INTEC & 48 & 52 & CPP-606-062 & 36.4 & 2000 & $\mathrm{O}_{2}$ Trim & \#2 Diesel & 216 & 32.3 & $\mathrm{Dc}$ & NA & Yes & Yes \\
\hline INTEC & 48 & 52 & СРP-606-063 & 36.4 & 2000 & $\mathrm{O}_{2}$ Trim & \#2 Diesel & 216 & 32.3 & $\mathrm{Dc}$ & NA & Yes & Yes \\
\hline INTEC & 48 & 52 & CPP-606-064 & 36.4 & 2000 & $\mathrm{O}_{2}$ Trim & \#2 Diesel & 216 & 32.3 & $\mathrm{Dc}$ & NA & Yes & Yes \\
\hline $\mathrm{NRF}^{\mathrm{b}}$ & 100 & $\theta$ & $\begin{array}{l}\text { NRF-620-014 } \\
\text { Boiler No.1 }\end{array}$ & 52.4 & 1961 & $\theta_{z}$ Trim & \#2 Diesel & 470 & 107 & NA & 2018 & Yes & Yes \\
\hline NRF & 100 & 0 & Boiler No. 4 & 29.3 & 2016 & $\mathrm{O}_{2}$ Trim & \#2 Diesel & 209 & 209 & $\mathrm{Dc}$ & NA & Yes & Yes \\
\hline NRF & 100 & $\theta$ & $\begin{array}{l}\text { NRF-620-012 } \\
\text { Boiler } \mathrm{N}_{0.3}\end{array}$ & 52.4 & 1961 & $\theta_{z}$ Trim & \#2 Diesel & 470 & 97.2 & NA & 2018 & Yes & Yes \\
\hline $\mathrm{NRF}^{\mathrm{a}}$ & 100 & 0 & Boiler No. 5 & 29.3 & 2017 & $\mathrm{O}_{2}$ Trim & \#2 Diesel & 209 & 209 & $\mathrm{Dc}$ & NA & No & No \\
\hline SMC & 90 & 10 & TAN 679-067a & 25 & 1987 & $\mathrm{O}_{2}$ Trim & \#2 Diesel & 167.5 & 35.4 & NA & NA & Yes & Yes \\
\hline SMC & 90 & 10 & TAN 679-068 & 25 & 1987 & $\mathrm{O}_{2}$ Trim & \#2 Diesel & 167.5 & 41.4 & NA & NA & Yes & Yes \\
\hline \multicolumn{14}{|c|}{ Notes: a. Green highlighted entries indicate boilers added after permit issuance. } \\
\hline & & & tris s with str & rough to & riocindi & hrilorc & fror & rvice. & & & & & \\
\hline
\end{tabular}

